\title{
Responses of terrestrial ecosystems to temperature and precipitation change: a meta-analysis of experimental manipulation
}

\author{
ZHUOTING WU*, PAUL DIJKSTRA*, GEORGE W. KOCH*, JOSEP PENUELAS† and \\ BRUCE A. HUNGATE* \\ *Department of Biological Sciences and Merriam-Powell Center for Environmental Research, Northern Arizona University, \\ Flagstaff. AZ 86011, USA, †Global Ecology Unit CSIC-CEAB-CREAF, CREAF (Centre de Recerce Ecologia i Apicacios Forestals), \\ Edifici C, Universitat Autònoma de Barcelona, 08193 Bellaterra, Barcelona, Spain
}

\begin{abstract}
Global mean temperature is predicted to increase by $2-7^{\circ} \mathrm{C}$ and precipitation to change across the globe by the end of this century. To quantify climate effects on ecosystem processes, a number of climate change experiments have been established around the world in various ecosystems. Despite these efforts, general responses of terrestrial ecosystems to changes in temperature and precipitation, and especially to their combined effects, remain unclear. We used metaanalysis to synthesize ecosystem-level responses to warming, altered precipitation, and their combination. We focused on plant growth and ecosystem carbon (C) balance, including biomass, net primary production (NPP), respiration, net ecosystem exchange (NEE), and ecosystem photosynthesis, synthesizing results from 85 studies. We found that experimental warming and increased precipitation generally stimulated plant growth and ecosystem $\mathrm{C}$ fluxes, whereas decreased precipitation had the opposite effects. For example, warming significantly stimulated total NPP, increased ecosystem photosynthesis, and ecosystem respiration. Experimentally reduced precipitation suppressed aboveground NPP (ANPP) and NEE, whereas supplemental precipitation enhanced ANPP and NEE. Plant productivity and ecosystem $\mathrm{C}$ fluxes generally showed higher sensitivities to increased precipitation than to decreased precipitation. Interactive effects of warming and altered precipitation tended to be smaller than expected from additive, single-factor effects, though low statistical power limits the strength of these conclusions. New experiments with combined temperature and precipitation manipulations are needed to conclusively determine the importance of temperature-precipitation interactions on the $\mathrm{C}$ balance of terrestrial ecosystems under future climate conditions.
\end{abstract}

Keywords: ecosystem photosynthesis, meta-analysis, net ecosystem exchange, plant biomass, plant productivity, precipitation, respiration, warming

Received 18 March 2010 and accepted 28 June 2010

\section{Introduction}

Increasing greenhouse gas emissions are expected to raise global mean temperature by $2-7^{\circ} \mathrm{C}$ by the end of this century (Allison et al., 2009). Precipitation is projected to increase at high latitudes and decrease in most subtropical regions (IPCC, 2007). Temperature and precipitation are key drivers of ecosystem processes, so projected climate changes will likely alter ecosystem carbon (C) balance. Understanding the sensitivity of terrestrial $\mathrm{C}$ balance to climate change is a high priority, because of the potential for changes in terrestrial $C$ storage to affect the pace of ongoing climatic change (Cox et al., 2000).

Correspondence: Zhuoting Wu, tel. + 1928523 2381, fax + 1928 523 7500, e-mail: zw27@nau.edu
While both ecosystem photosynthesis and respiration often increase with warming (Rustad et al., 2001), responses of net $\mathrm{C}$ balance to warming are less clear. Some lines of evidence suggest that warming increases net $\mathrm{C}$ uptake (Welker et al., 2004; Oberbauer et al., 2007; Sullivan et al., 2008). Also, part of the residual terrestrial $\mathrm{C}$ sink is attributed to recent warming and lengthening of the growing season (Peñuelas \& Filella, 2001; Lucht et al., 2002; Nemani et al., 2003). Furthermore, global C cycle models project increased terrestrial $\mathrm{CO}_{2}$ uptake in response to warming through the middle of this century (Cao \& Woodward, 1998; Cox et al., 2000; Cramer et al., 2001; Fung et al., 2005; Friedlingstein et al., 2006; Canadell et al., 2007; Sitch et al., 2008). On the other hand, the strong sensitivity of respiration to warming provides a potential positive feedback to warming (Woodwell et al., 1998; Knorr et al., 2005; Heimann \& Reichstein, 2008), 
and suggests that the sensitivity of respiration to warming will eventually surpass that of ecosystem photosynthesis. This is consistent with projections of most global biogeochemical models that continued warming will cause declines in net $\mathrm{C}$ uptake by around midcentury (Cao \& Woodward, 1998; Cox et al., 2000; Cramer et al., 2001; Fung et al., 2005; Friedlingstein et al., 2006; Canadell et al., 2007). Results from field experiments can help shed light on the direction and magnitude of ecosystem $C$ balance responses to warming. Our first goal in this work was to synthesize results from field experiments that have examined the responses of plant biomass, productivity, and $\mathrm{C}$ balance of terrestrial ecosystems to experimental warming.

Water availability is critical to all life, so altered precipitation is virtually certain to affect terrestrial ecosystem processes. Yet, most inferences about the effects of altered precipitation on ecosystem processes rely on correlations between interannual and intersite variation in precipitation and processes of interest (Lieth, 1973; Churkina et al., 1999; Knapp \& Smith, 2001; Scurlock et al., 2002; Huxman et al., 2004; Garbulsky et al., 2010). This approach takes advantage of the natural variation in precipitation between years, yet correlations have the potential drawback of confounding changes in other variables that covary with precipitation over space and time, such as temperature (e.g., Loik et al., 2004; Breshears et al., 2005; Ciais et al., 2005). Experimental manipulations of precipitation can complement, and extend beyond, observational studies by causally linking precipitation change with ecosystem responses while keeping covarying climate variables constant. A number of field experiments examining responses of $\mathrm{C}$ cycling to altered precipitation now exist. Our second goal in this work was to synthesize results from these experiments using meta-analysis.

The combined effects of warming and altered precipitation are expected to have strong influences on $\mathrm{C}$ balance. For example, the combination of warming and decreased precipitation can cause large $C$ losses (Loik et al., 2004; Angert et al., 2005; Breshears et al., 2005; Ciais et al., 2005). The expected shift from terrestrial C sink to source could be hastened if decreased precipitation occurs along with warming in the next few decades. Yet, warming-induced soil drying can also suppress soil respiration and thereby increase net $C$ storage (Saleska et al., 2003). A few field experiments have examined the interactive effects of warming and altered precipitation on $\mathrm{C}$ balance. Our third goal was to synthesize these interactive effects using meta-analysis.

Different terrestrial ecosystems are likely to vary in the magnitude and direction of their responses to warming and altered precipitation. For example, aboveground net primary production (ANPP) in cooler eco- systems has been found to exhibit stronger positive responses to warming than that of warmer ecosystems (Rustad et al., 2001). Meanwhile, more C was lost in colder ecosystems due to a higher sensitivity of soil respiration to warming (Kirschbaum, 1995), which could potentially render such ecosystems as C sources. Reduced precipitation may have disproportionately large impacts on the $\mathrm{C}$ balance of semiarid ecosystems compared with ecosystems in more mesic environments. There have been a number of field experiments addressing effects of warming and altered precipitation on components of $\mathrm{C}$ balance, crossing a broad spectrum of climatic space. Our fourth goal was to test whether different ecosystem types and biomes, representing climatic space, vary systematically in their responses to warming and altered precipitation.

Meta-analysis provides a synthesis of individual studies and allows statistical testing whether responses are general across a variety of sites and conditions. For example, Arft et al. (1999) applied meta-analysis to examine the response of plant phenology, growth, and reproduction to experimental warming using 13 circumpolar experimental sites. Rustad et al. (2001) also used meta-analysis to synthesize findings on the responses of soil respiration, net nitrogen mineralization, and aboveground plant growth to experimental warming from 32 ecosystem warming experiments. In this paper, we applied meta-analysis to synthesize responses of plant biomass, productivity, and ecosystem $\mathrm{C}$ balance to warming and altered precipitation. We asked the following questions: (1) how do warming, increased and decreased precipitation, acting in isolation, affect plant growth and ecosystem C fluxes? (2) how do these responses vary across vegetation types and with climate?, and (3) is there evidence for interactive effects between warming and altered precipitation on plant growth and C cycling? We synthesized data from 85 studies where temperature, precipitation, or both were manipulated, covering tundra, boreal forests, temperate evergreen and deciduous forests, shrublands, grasslands, and deserts (Fig. 1 and Table 1).

\section{Methods}

\section{Data compilation}

Criteria for study selection influence the output of metaanalysis (Hungate et al., 2009). Our criteria were: for multiple observations from a single site, we included the most recent result, because no significant temporal patterns were observed in the responses of soil respiration and plant productivity to warming in a previous meta-analysis (Rustad et al., 2001); for multifactor experiments (e.g., warming and nutrient), we used data from temperature and/or precipitation treatments while 


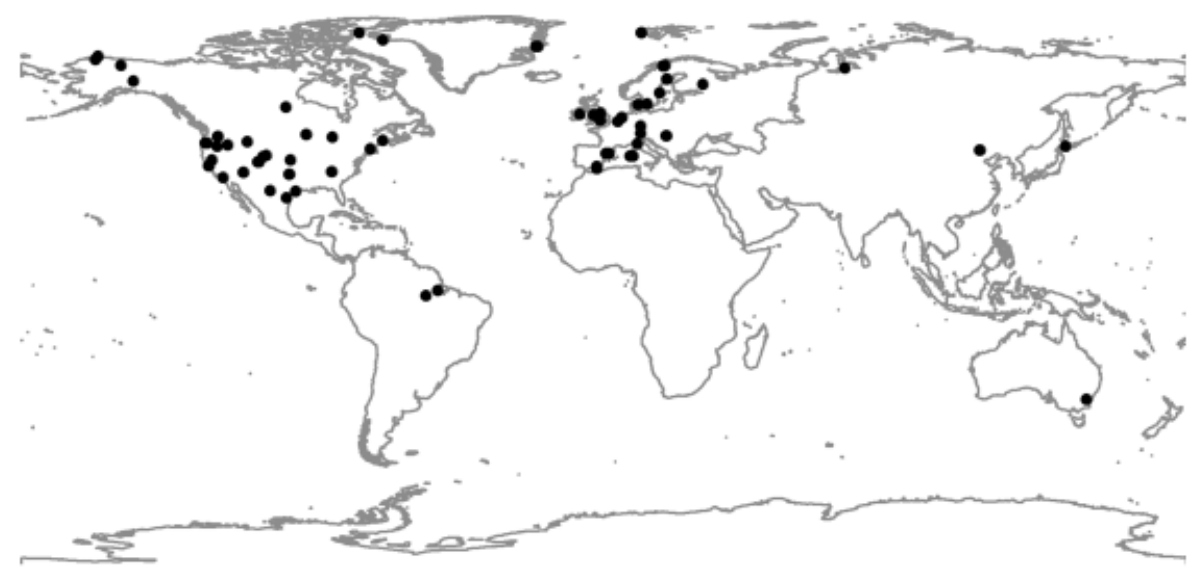

Fig. 1 Study sites included in this meta-analysis.

the other factors were kept at ambient levels (e.g., warming at ambient nutrient conditions), and interactive responses when warming and altered precipitation were combined factorially; we treated multiple levels of treatment as independent, even though they shared a common control. We used Google Scholar (Google Inc., Mountain View, CA, USA) for exhaustive search of peer-reviewed journal articles published before June 2009. Data collection was restricted to studies that were conducted in the field; no laboratory incubation or growth chamber experiments were included. For each selected study, we collected latitude, longitude, elevation, mean annual temperature (MAT) and precipitation (MAP), and biome type; we also collected information on experimental duration, soil moisture and temperature, experimental treatment type and size, treatment techniques, control and treatment means, sample size, and variance. These studies were located from $35.12 \mathrm{~S}$ to 78.56 $\mathrm{N}$, with MAT of experimental sites ranging from -20 to $25^{\circ} \mathrm{C}$, and MAP from 200 to $2272 \mathrm{~mm}$. We also grouped these studies into two broad vegetation types - woody and herbaceous - to test for differences in responses of functional groups. The experimental duration ranged from 1 to 11 years. Warming techniques included curtain covers, heating cables, open or closed top chambers, greenhouses, overhead infrared heat lamps, transplanting and passive nighttime warming; altered precipitation was achieved by hand sprinklers, metered hand sprayers, rain collectors, rainout shelters, sheeting, guttering, and pumping. Warming treatments caused an increase in soil temperature ranging from 0.1 to $10.2^{\circ} \mathrm{C}$; decreased precipitation treatments were between 41 and $1136 \mathrm{~mm}$, and increased precipitation treatments ranged from 5 to $2148 \mathrm{~mm}$.

We grouped the collected data into five categories of response variables (Table 2). (1) Biomass: For herbaceous plants, aboveground biomass was measured by clipping live biomass at the soil surface, oven-drying, and weighing. Allometric relationships were used for estimating woody plant aboveground biomass. Belowground biomass was measured by taking soil cores up to $30 \mathrm{~cm}$ in depth, removing plant tissues, oven-drying, and weighing. Total biomass was obtained from the sum of the aboveground and belowground biomass. (2) Net primary productivity (NPP): ANPP was calculated by peak aboveground biomass when there is no carryover of living biomass from previous years. Canopy biomass and shoot mass/shoot length relationships were also used for calculating ANPP of woody plants. Belowground NPP (BNPP) was estimated using root ingrowth cores, root distribution regression, and root biomass/root turnover rate relationships. Total NPP (TNPP) was calculated as the sum of ANPP and BNPP. (3) Respiration: Ecosystem respiration was obtained by measuring $\mathrm{CO}_{2}$ exchange in the dark (either covering the gas-exchange chamber with shade cloth or measuring at night), using infrared gas analyzer (IRGA) or periodic headspace sampling and gas chromatography. Soil respiration was measured by IRGA or headspace sampling followed by gas chromatography. Aboveground respiration was calculated by subtracting soil respiration from ecosystem respiration. (4) Net ecosystem exchange (NEE): NEE was measured using a transparent chamber with an IRGA. (5) Ecosystem photosynthesis: Ecosystem photosynthesis was calculated by the sum of NEE and ecosystem respiration.

\section{Meta-analysis}

Effect sizes. Effect size compares the treatment effects of all studies and expresses them on a common scale, aiming to highlight general responses over a broad range of ecosystems. A number of different metrics can be used for meta-analysis (Rosenberg et al., 2000; Hungate et al., 2009). For each response variable, we calculated three types of effect size metrics.

$$
\text { Log response ratio : } X_{\mathrm{LR}}=\ln (T / C) \text {, }
$$

where $T$ and $C$ are the means of treatment and control groups, respectively. The log ratio compares the relative difference between the treatments and controls.

$$
\text { Absolute difference : } X_{\mathrm{AD}}=T-C \text {, }
$$

where $T$ and $C$ are defined as above. For biogeochemistry, this metric is effective in expressing ecological significance because it captures the magnitude of changes in mass or mass fluxes.

$$
\begin{aligned}
\text { Sensitivity }: & X_{\mathrm{S}}=(T-C) /\left(T_{\mathrm{T}}-T_{\mathrm{C}}\right) \\
& \text { or } X_{\mathrm{S}}=(T-C) /\left(\mathrm{PPT}_{\mathrm{T}}-\mathrm{PPT}_{\mathrm{C}}\right),
\end{aligned}
$$


Table 1 Site characteristics for temperature and precipitation manipulation studies included in this meta-analysis

\begin{tabular}{|c|c|c|c|c|c|c|}
\hline State/country & Site & Latitude & Longitude & Biome & Period & References \\
\hline \multicolumn{7}{|c|}{ Temperature manipulation experiments } \\
\hline Alaska, USA & Delta Junction & 63.55 & -145.44 & Boreal forest & 2000-2007 & $\begin{array}{l}\text { Bergner et al. (2004), Allisor } \\
\quad \text { \& Treseder (2008) }\end{array}$ \\
\hline Alaska, USA & Toolik Lake & 68.38 & -149.34 & Tundra & 1989-2002 & $\begin{array}{l}\text { Hobbie \& Chapin (1998), } \\
\text { Jones et al. (1998), } \\
\text { Grogan and Chapin III } \\
\text { (2000), Welker } \text { et al. } \\
\text { (1999), Oberbauer et al. } \\
\text { (2007), La Puma et al. } \\
\text { (2007) }\end{array}$ \\
\hline Alaska, USA & Atqasuk & 70.27 & -157.24 & Tundra & 1996-2001 & Oberbauer et al. (2007) \\
\hline Alaska, USA & Barrow & 71.18 & -156.4 & Tundra & 1995-2001 & Oberbauer et al. (2007) \\
\hline Australia & $\begin{array}{l}\text { Ginninderra } \\
\text { Experiment Station }\end{array}$ & -35.12 & 149.06 & Grassland & 1995 & Lilley et al. (2001) \\
\hline Austria & $\begin{array}{l}\text { Northern Limestone } \\
\text { Alps }\end{array}$ & 47.35 & 11.38 & Forest & 2004-2006 & Schindlbacher et al. (2009) \\
\hline Belgium & Drie Eiken Campus & 51.09 & 4.24 & Grassland & 2003-2005 & De Boeck et al. $(2007,2008)$ \\
\hline California, USA & $\begin{array}{l}\text { Jasper Ridge } \\
\text { Biological Preserve }\end{array}$ & 37.24 & -122.14 & Grassland & 1997-2003 & $\begin{array}{l}\text { Zavaleta et al. (2003), } \\
\text { Dukes et al. (2005) }\end{array}$ \\
\hline Canada & Alexandra Fiord & 78.53 & -75.55 & Tundra & 1992-2001 & $\begin{array}{l}\text { Welker et al. (2004), } \\
\quad \text { Oberbauer et al. (2007) }\end{array}$ \\
\hline Canada & $\begin{array}{l}\text { Boreal Soil and Air } \\
\text { Warming } \\
\text { Experiment } \\
\text { research site }\end{array}$ & 55.53 & -98.2 & $\begin{array}{l}\text { Black spruce } \\
\text { forest }\end{array}$ & 2004-2005 & Bronson et al. (2008) \\
\hline Oregon, USA & $\begin{array}{l}\text { National Health and } \\
\text { Environmental } \\
\text { Effects Research } \\
\text { Laboratory }\end{array}$ & 44.34 & -123.17 & Douglas fir & 1993-1997 & $\begin{array}{l}\text { Olszyk et al. (2003), Tingey } \\
\quad \text { et al. (2007) }\end{array}$ \\
\hline China & Duolun County & 42.02 & 116.17 & Steppe & 2005-2008 & $\begin{array}{l}\text { Niu et al. (2008), Liu et al. } \\
\text { (2009), Xia et al. (2009) }\end{array}$ \\
\hline Colorado, USA & Niwot Ridge & 40.03 & -105.36 & Tundra & 1994-1997 & Welker et al. (1999) \\
\hline Colorado, USA & $\begin{array}{c}\text { Rocky Mountain } \\
\text { Biological } \\
\text { Laboratory }\end{array}$ & 38.53 & -107.02 & $\begin{array}{l}\text { Montane } \\
\text { meadow }\end{array}$ & 1991-1997 & $\begin{array}{l}\text { Harte \& Shaw (1995), De } \\
\text { Valpine \& Harte (2001), } \\
\text { Saleska et al. }(2002)\end{array}$ \\
\hline Denmark & Mols CLIMOOR & 56.23 & 10.57 & Shrub & 1999-2001 & Emmett et al. (2004) \\
\hline Finland & $\begin{array}{l}\text { Mekrijarvi Research } \\
\text { Station } \\
\text { (University of } \\
\text { Joensuu) }\end{array}$ & 62.47 & 30.58 & $\begin{array}{l}\text { Scots pine } \\
\text { forest }\end{array}$ & 1996-2000 & Niinistö et al. (2004) \\
\hline Greenland & Pituffik & 76.33 & -68.3 & $\begin{array}{l}\text { High arctic } \\
\text { fen }\end{array}$ & 2003-2006 & Sullivan et al. (2008) \\
\hline Greenland & $\begin{array}{l}\text { Zackenberg } \\
\quad \text { Research Station }\end{array}$ & 74.28 & -20.34 & Grassland & 1998-1999 & $\begin{array}{l}\text { Mertens et al. (2001), } \\
\quad \text { Marchand et al. (2004) }\end{array}$ \\
\hline Hungary & VULCAN & 46.53 & 19.23 & Shrub & 2002-2005 & Peñuelas et al. (2007) \\
\hline Hungary & Danube Tisza & 46.52 & 19.25 & Forest-steppe & 2002-2006 & Lellei-Kovács et al. (2008) \\
\hline Italy & Sardinia VULCAN & 40.36 & 8.9 & Shrub & 2004 & Peñuelas et al. (2007) \\
\hline Japan & Taisetsu Mountains & 43.33 & 142.53 & Shrub & 1994-1999 & Kudo \& Suzuki (2003) \\
\hline Maine, USA & $\begin{array}{l}\text { Howland Integrated } \\
\text { Forest Study }\end{array}$ & 45.1 & -68.4 & $\begin{array}{l}\text { Spruce-fir } \\
\text { forest }\end{array}$ & 1993-1995 & Rustad \& Fernandez (1998) \\
\hline $\begin{array}{l}\text { Massachusetts, } \\
\text { USA }\end{array}$ & Harvard Forest & 42.54 & -72.18 & $\begin{array}{l}\text { Hardwood } \\
\text { forest }\end{array}$ & 1991-2000 & Melillo et al. (2002) \\
\hline Minnesota, USA & $\begin{array}{l}\text { Glacial Lake Upham } \\
\text { basin (Toivola } \\
\text { and Alborn) }\end{array}$ & 47 & -92 & $\begin{array}{l}\text { Bog and } \\
\text { sedge fen }\end{array}$ & 1994-1997 & $\begin{array}{l}\text { Weltzin et al. (2000), } \\
\text { Updegraff et al. (2001) }\end{array}$ \\
\hline The Netherlands & $\begin{array}{l}\text { Oldebroek } \\
\quad \text { CLIMOOR }\end{array}$ & 52.24 & 5.55 & Shrub & 1999-2003 & $\begin{array}{l}\text { Emmett et al. (2004), } \\
\text { Peñuelas et al. (2007) }\end{array}$ \\
\hline Norway & Svalbard & 78.56 & 11.5 & Shrub & 1991-1993 & Wookey et al. (1995) \\
\hline Russia & Tazovskiy Peninsula & 67.56 & 74.52 & $\begin{array}{l}\text { Dwarf shrub } \\
\text { tundra }\end{array}$ & 2002-2003 & Biasi et al. (2008) \\
\hline
\end{tabular}


Table 1 (Contd.)

\begin{tabular}{|c|c|c|c|c|c|c|}
\hline State/country & Site & Latitude & Longitude & Biome & Period & References \\
\hline Oklahoma, USA & $\begin{array}{c}\text { Great Plain Apiaries } \\
\text { (Kessler's Farm } \\
\text { Field Laboratory) }\end{array}$ & 34.59 & -97.31 & Grassland & 1999-2003 & $\begin{array}{l}\text { Luo et al. (2001, 2009), Wan } \\
\text { et al. (2005), Zhou et al. } \\
\quad(2006,2007)\end{array}$ \\
\hline Italy & Capo Caccia & 40.37 & 8.1 & Forest & 2002-2004 & De Dato et al. (2006) \\
\hline Spain & $\begin{array}{l}\text { Catalonia } \\
\text { CLIMOOR }\end{array}$ & 41.18 & 1.49 & Shrub & 1999-2005 & $\begin{array}{l}\text { Emmett et al. (2004), } \\
\text { Peñuelas et al. (2007), } \\
\text { Sardans et al. (2008) }\end{array}$ \\
\hline Sweden & Abisko & 68.19 & 18.51 & $\begin{array}{c}\text { Subarctic } \\
\text { heath }\end{array}$ & 1989-1999 & $\begin{array}{l}\text { Jonasson et al. (1999), Illeris } \\
\text { et al. (2004) }\end{array}$ \\
\hline Sweden & Abisko & 68.21 & 18.49 & Bog & 2000-2002 & Dorrepaal et al. (2004) \\
\hline Sweden & $\begin{array}{l}\text { Abisko Scientific } \\
\text { Research Station }\end{array}$ & 68.35 & 18.82 & $\begin{array}{r}\text { Subarctic } \\
\text { dwarf } \\
\text { shrub } \\
\text { heath }\end{array}$ & 1991-1997 & $\begin{array}{l}\text { Press et al. (1998), Hartley } \\
\quad \text { et al. (1999) }\end{array}$ \\
\hline Sweden & Degero Stormyr & 64.11 & 19.33 & Fen & 1995-1998 & Gunnarsson et al. (2004) \\
\hline Sweden & $\begin{array}{l}\text { Latnjajaure field } \\
\text { station }\end{array}$ & 68.21 & 18.21 & $\begin{array}{l}\text { Mesic sedge } \\
\text { meadow }\end{array}$ & 1994-1998 & Jónsdóttir et al. (2005) \\
\hline Sweden & Lappmyran & 64.09 & 19.35 & Bog & 2004-2005 & Breeuwer et al. (2008) \\
\hline Sweden & Åkerlänna Römosse & 60.01 & 17.22 & Bog & 2004-2005 & Breeuwer et al. (2008) \\
\hline Sweden & Saxnäs Mosse & 56.51 & 13.27 & Bog & $2004-2005$ & Breeuwer et al. (2008) \\
\hline Tennessee, USA & $\begin{array}{l}\text { Oak Ridge National } \\
\text { Laboratory } \\
\text { Global Change } \\
\text { Field Research } \\
\text { Facility }\end{array}$ & 35.54 & -84.2 & Grassland & 2002-2004 & Wan et al. (2007) \\
\hline Tennessee, USA & $\begin{array}{l}\text { Oak Ridge National } \\
\text { Laboratory } \\
\text { Global Change } \\
\text { Field Research } \\
\text { Facility }\end{array}$ & 35.54 & -84.2 & $\begin{array}{l}\text { Red maple } \\
\text { and sugar } \\
\text { maple } \\
\text { seedlings }\end{array}$ & 1994-1997 & Wan et al. (2004) \\
\hline United Kingdom & Buxton & 53.2 & -2 & $\begin{array}{l}\text { Limestone } \\
\text { grassland }\end{array}$ & 1994-2004 & Grime et al. $(2000,2008)$ \\
\hline United Kingdom & University of York & 53.58 & -1.06 & Wheat, maize & 2004-2005 & Hartley et al. (2007) \\
\hline United Kingdom & Wytham & 51.46 & -1.2 & $\begin{array}{l}\text { Limestone } \\
\text { grassland }\end{array}$ & $1994-1998$ & $\begin{array}{l}\text { Grime et al. (2000), } \\
\text { Thompson et al. (2000) }\end{array}$ \\
\hline United Kingdom & $\begin{array}{l}\text { Clocaenog } \\
\text { CLIMOOR }\end{array}$ & 53.03 & -3.28 & Shrub & 1999-2003 & $\begin{array}{l}\text { Emmett et al. (2004), } \\
\text { Peñuelas et al. (2007) }\end{array}$ \\
\hline \multicolumn{7}{|c|}{ Precipitation manipulation experiments } \\
\hline Brazil & Amazon & -2.9 & -54.95 & Forest & 2000-2004 & Davidson et al. (2008) \\
\hline Brazil & $\begin{array}{l}\text { Caxiuana National } \\
\text { Forest }\end{array}$ & -1.43 & -51.27 & Forest & $2002-2003$ & Sotta et al. (2007) \\
\hline California, USA & $\begin{array}{l}\text { Sierra Foothill } \\
\text { research and } \\
\text { Extension Center }\end{array}$ & 39.15 & -121.17 & Grassland & 2003-2006 & $\begin{array}{l}\text { Silver et al. (2005), Chou } \\
\text { et al. (2008) }\end{array}$ \\
\hline California, USA & $\begin{array}{l}\text { Jasper Ridge } \\
\text { Biological } \\
\text { Preserve }\end{array}$ & 37.24 & -122.14 & Grassland & 1997-2003 & $\begin{array}{l}\text { Zavaleta et al. (2003), Dukes } \\
\text { et al. (2005) }\end{array}$ \\
\hline California, USA & $\begin{array}{l}\text { Irvine Ranch Land } \\
\text { Reserve }\end{array}$ & 33.62 & -117.76 & Grassland & 2006 & Harpole et al. (2007) \\
\hline Spain & $\begin{array}{l}\text { Catalonia } \\
\text { CLIMOOR }\end{array}$ & 41.18 & 1.49 & Shrub & 1999-2005 & $\begin{array}{l}\text { Emmett et al. (2004), } \\
\text { Peñuelas et al. (2007) }\end{array}$ \\
\hline China & Duolun County & 42.02 & 116.16 & Steppe & 2005-2008 & $\begin{array}{l}\text { Xiao et al. (2007), Niu et al. } \\
\text { (2008), Chen et al. (2009), } \\
\text { Liu et al. (2009) }\end{array}$ \\
\hline China & Xilingol & $\begin{array}{l}43.26- \\
44.29\end{array}$ & $\begin{array}{l}115.32- \\
117.12\end{array}$ & Grassland & 2005 & Chen et al. (2008) \\
\hline Denmark & Mols CLIMOOR & 56.23 & 10.57 & Shrub & 1999-2001 & $\begin{array}{l}\text { Emmett et al. (2004), } \\
\text { Peñuelas et al. (2007) }\end{array}$ \\
\hline
\end{tabular}


Table 1 (Contd.)

\begin{tabular}{|c|c|c|c|c|c|c|}
\hline State/country & Site & Latitude & Longitude & Biome & Period & References \\
\hline Germany & $\begin{array}{l}\text { Ecological-botanical } \\
\text { Garden of } \\
\text { Bayreuth } \\
\text { University }\end{array}$ & 49.55 & 11.35 & Grassland & 2005 & Mirzaei et al. (2008) \\
\hline Greenland & $\begin{array}{l}\text { Zackenberg } \\
\quad \text { Research Station }\end{array}$ & 74.3 & -21 & $\begin{array}{l}\text { High arctic } \\
\text { semi } \\
\text { desert }\end{array}$ & 1997-1999 & Illeris et al. (2003) \\
\hline Hungary & DanubeTisza & 46.52 & 19.25 & Forest-steppe & 2002-2006 & Lellei-Kovács et al. (2008) \\
\hline Hungary & CLIMOOR & 46.53 & 19.23 & Shrub & 2002-2005 & Peñuelas et al. (2007) \\
\hline Ireland & Clara bog & 53.19 & -7.58 & Bog & 2007 & Robroek et al. (2009) \\
\hline Italy & Monte Rondinaio & 44.08 & 10.35 & $\begin{array}{l}\text { Dwarf-shrub } \\
\text { heath }\end{array}$ & 1999-2003 & Lisa et al. (2007) \\
\hline Kansas, USA & $\begin{array}{l}\text { Konza Prairie } \\
\text { Biological Station }\end{array}$ & 39.1 & -96.9 & $\begin{array}{l}\text { Tallgrass } \\
\text { prairie }\end{array}$ & 2004 & $\begin{array}{l}\text { Fay et al. }(2000,2008) \\
\text { Harper et al. }(2005)\end{array}$ \\
\hline The Netherlands & $\begin{array}{l}\text { Oldebroek } \\
\text { CLIMOOR }\end{array}$ & 52.24 & 5.55 & Shrub & 1999-2003 & $\begin{array}{l}\text { Emmett et al. (2004), } \\
\text { Peñuelas et al. (2007) }\end{array}$ \\
\hline Norway & Svalbard & 78.56 & 11.5 & Shrub & 1991-1993 & Wookey et al. (1995) \\
\hline United Kingdom & $\begin{array}{l}\text { Clocaenog } \\
\text { CLIMOOR }\end{array}$ & 53.03 & -3.28 & Shrub & 1999-2001 & $\begin{array}{l}\text { Emmett et al. (2004), } \\
\quad \text { Peñuelas et al. (2007) }\end{array}$ \\
\hline Oklahoma, USA & $\begin{array}{l}\text { Great Plain Apiaries } \\
\text { (Kessler's Farm } \\
\text { Field Laboratory) }\end{array}$ & 34.59 & -97.31 & Grassland & $2002-2003$ & $\begin{array}{l}\text { Zhou et al. (2006), Sherry } \\
\text { et al. (2008) }\end{array}$ \\
\hline Oregon, USA & $\begin{array}{l}\text { Northern Great } \\
\text { Basin } \\
\text { Experimental } \\
\text { Range }\end{array}$ & 43.29 & -119.43 & Grassland & 1994-2000 & Bates et al. (2006) \\
\hline Italy & Capo Caccia & 40.37 & 8.1 & Forest & 2002-2004 & De Dato et al. (2006) \\
\hline Italy & Sardinia VULCAN & 40.36 & 8.9 & Shrub & 2004 & Peñuelas et al. (2007) \\
\hline Spain & $\begin{array}{l}\text { Prades Mountains in } \\
\text { Southern } \\
\text { Catalonia }\end{array}$ & 41.13 & 0.55 & $\begin{array}{l}\text { Holm oak } \\
\text { forest }\end{array}$ & 1999-2003 & Ogaya \& Peñuelas (2007) \\
\hline Spain & Cabo de Gata & 36.49 & -2.15 & $\begin{array}{c}\text { Shortgrass } \\
\text { prairie }\end{array}$ & 2005-2006 & Miranda et al. (2009) \\
\hline Spain & El Cautivo & 37 & -2.26 & $\begin{array}{c}\text { Shortgrass } \\
\text { prairie }\end{array}$ & 2005-2006 & Miranda et al. (2009) \\
\hline Spain & $\begin{array}{l}\text { Catalonia } \\
\text { CLIMOOR }\end{array}$ & 41.18 & 1.49 & Shrub & 1999-2005 & Sardans et al. (2008) \\
\hline Sweden & $\begin{array}{l}\text { Abisko Scientific } \\
\text { Research Station }\end{array}$ & 68.21 & 18.49 & Shrub & 1991-1995 & Press et al. (1998) \\
\hline Texas, USA & $\begin{array}{l}\text { The University of } \\
\text { Houston Coastal } \\
\text { Center }\end{array}$ & 29.38 & -95.04 & $\begin{array}{l}\text { Tallgrass } \\
\text { prairie }\end{array}$ & 2002-2004 & Siemann et al. (2007) \\
\hline Texas, USA & $\begin{array}{l}\text { Big Bend National } \\
\text { Park }\end{array}$ & 29.5 & -103.1 & Grassland & 2002-2004 & Patrick et al. (2007) \\
\hline Texas, USA & $\begin{array}{l}\text { Texas Agricultural } \\
\text { Experiment } \\
\text { Station }\end{array}$ & 27.4 & -98.12 & Grassland & 1996-1997 & McCulley et al. (2007) \\
\hline United Kingdom & Buxton & 53.2 & -2 & $\begin{array}{l}\text { Limestone } \\
\text { grassland }\end{array}$ & 1994-1998 & Grime et al. $(2000,2008)$ \\
\hline United Kingdom & Wytham & 51.46 & -1.2 & $\begin{array}{l}\text { Limestone } \\
\text { grassland }\end{array}$ & 1994-1998 & Grime et al. $(2000,2008)$ \\
\hline Wyoming, USA & $\begin{array}{l}\text { Yellowstone } \\
\text { National Park }\end{array}$ & $44.55-45.1$ & $\begin{array}{l}-110.1 \text { to } \\
-110.5\end{array}$ & Grassland & 2005 & Risch \& Frank (2007) \\
\hline \multicolumn{7}{|c|}{ Temperature $\times$ precipitation manipulation experiments } \\
\hline California, USA & $\begin{array}{c}\text { Jasper Ridge } \\
\text { Biological } \\
\text { Preserve }\end{array}$ & 37.24 & -122.14 & Grassland & 1998-2003 & Dukes et al. (2005) \\
\hline
\end{tabular}


Table 1 (Contd.)

\begin{tabular}{|c|c|c|c|c|c|c|}
\hline State/country & Site & Latitude & Longitude & Biome & Period & References \\
\hline Arizona, USA & $\begin{array}{l}\text { C. Hart Merriam } \\
\text { elevation } \\
\text { gradient }\end{array}$ & $\begin{array}{l}35.35- \\
35.69\end{array}$ & $\begin{array}{l}-111.43 \text { to } \\
-111.73\end{array}$ & Grassland & 2002-2009 & $\begin{array}{l}\text { Z. Wu, P. Dijkstra, G. W. } \\
\text { Koch, B. A. Hungate, } \\
\text { unpublished results }\end{array}$ \\
\hline China & Duolun County & 42.02 & 116.17 & Steppe & 2005-2008 & $\begin{array}{l}\text { Niu et al. (2008), Liu et al. } \\
\text { (2009) }\end{array}$ \\
\hline Oklahoma, USA & $\begin{array}{l}\text { Great Plain Apiaries } \\
\text { (Kessler's Farm } \\
\text { Field Laboratory) }\end{array}$ & 34.59 & -97.31 & $\begin{array}{l}\text { Tallgrass } \\
\text { prairie }\end{array}$ & 2002-2004 & $\begin{array}{l}\text { Zhou et al. (2006), Sherry } \\
\text { et al. (2008) }\end{array}$ \\
\hline United Kingdom & Buxton & 53.2 & -2 & $\begin{array}{l}\text { Limestone } \\
\text { grassland }\end{array}$ & 1994-2004 & $\begin{array}{l}\text { Grime et al. }(2000,2008), \\
\text { Thompson et al. }(2000)\end{array}$ \\
\hline United Kingdom & Wytham & 51.46 & -1.2 & $\begin{array}{l}\text { Limestone } \\
\text { grassland }\end{array}$ & 1994-1998 & $\begin{array}{l}\text { Grime et al. (2000, 2008), } \\
\text { Thompson et al. (2000) }\end{array}$ \\
\hline
\end{tabular}

Table 2 Response variables investigated in the meta-analysis

\begin{tabular}{l}
\hline 1. Biomass $\left(\mathrm{g} \mathrm{m}^{-2}\right)$ \\
a. Total biomass \\
b. Aboveground biomass \\
c. Belowground biomass \\
2. Net primary productivity $\left(\mathrm{NPP}, \mathrm{g} \mathrm{m}^{-2} \mathrm{yr}^{-1}\right)$ \\
a. Total NPP \\
b. Aboveground NPP \\
c. Belowground NPP \\
3. Respiration ( $\mu$ mol $\left.\mathrm{CO}_{2} \mathrm{~m}^{-2} \mathrm{~s}^{-1}\right)$ \\
a. Ecosystem respiration \\
b. Aboveground respiration \\
c. Soil respiration \\
4. Net ecosystem exchange $\left(\mu \mathrm{mol} \mathrm{CO}_{2} \mathrm{~m}^{-2} \mathrm{~s}^{-1}\right)$ \\
5. Ecosystem photosynthesis $\left(\mu \mathrm{mol} \mathrm{CO}_{2} \mathrm{~m}^{-2} \mathrm{~s}^{-1}\right)$
\end{tabular}

where $T$ and $C$ are defined as above, $T_{\mathrm{T}}$ and $T_{\mathrm{C}}$ are the soil temperature in treatment and control plots, respectively, and $\mathrm{PPT}_{\mathrm{T}}$ and $\mathrm{PPT}_{\mathrm{C}}$ are the precipitation amounts received in treatment and control plots, respectively. This metric was used to quantify responses to climate change treatments, normalizing absolute responses to the magnitude of the treatment imposed. This metric yields positive values if the response is in the same direction as the climate change treatment. For example, in ecosystems where water is a limiting resource for plant growth, reduced biomass in response to decreased precipitation treatment and increased biomass in response to supplemental precipitation both yield positive values of sensitivity, facilitating the comparison of experiments where treatment size or direction of precipitation manipulation differed. We also used the sensitivity metrics to compare the magnitude of responses to decreased and increased precipitation treatments.

Weighting functions. We tested the sensitivity of the results to various weighting functions, using weights based on equal weights, variance, sample size, and experimental duration. We applied four different weighting functions:
(1) Weighting all the studies uniformly, where effect size metrics only depend on the means of control and treatment groups.

(2) Weighting by the inverse of the pooled variance (Hedges \& Olkin, 1985), such that studies with lower variance have higher weight.

(3) Weighting by sample size as calculated from

$$
\text { weight }_{N}=\left(N_{\mathrm{C}} N_{\mathrm{T}}\right) /\left(N_{\mathrm{C}}+N_{\mathrm{T}}\right)
$$

where $N_{\mathrm{C}}$ and $N_{\mathrm{T}}$ are the sample sizes for control and treatment groups, respectively. More weights are given to well-replicated studies with larger sample sizes.

(4) Weighting by experimental duration as calculated from

$$
\text { weight }_{d}=\left(d_{\mathrm{C}} d_{\mathrm{T}}\right) /\left(d_{\mathrm{C}}+d_{\mathrm{T}}\right),
$$

where $d_{\mathrm{C}}$ and $d_{\mathrm{T}}$ are the experimental durations of the control and treatment groups, respectively. This approach gives higher weights to experiments that last longer.

We used METAWIN 2.1 (Rosenberg et al., 2000) to conduct metaanalyses, generating mean effect sizes and $95 \%$ bootstrapped confidence intervals $(95 \% \mathrm{CI})$. Treatment effects are considered significant when the $95 \% \mathrm{CI}$ does not overlap with 0 , and the direction and magnitude of the effect are determined by the sign and size of the $95 \%$ CI. We present summary results for $\log$ ratio effect sizes weighted by all weighting functions, but for absolute and sensitivity metrics we chose to present results weighted by sample size, because different weighing functions had little influence on the significance of results. In the text, we report mean responses to climate change treatments, and their 95\% CIs, to capture the general pattern and magnitude of the influence of climate change treatments, as well as the range observed. For efficiency, we report mean responses and 95\% CIs as: mean value (lower confidence limit to upper confidence limit) and appropriate units. This convention will be used throughout this article.

\section{Statistical analysis}

Tests of interactions. To evaluate the interactive effects of temperature and precipitation, for each experiment, we calculated the expected interactive response based on 
observed single factor responses, assuming that effects were additive, and compared the expected against the observed interactive effect. Sufficient sample size for this analysis $(n>2)$ existed for aboveground biomass, ANPP, ecosystem respiration, NEE, and ecosystem photosynthesis, but not for belowground and total plant biomass and productivity, aboveground and soil respiration. We used $\mathrm{R}$ to test whether the slope differed from 1 and intercept from 0 in the linear regression $(\mathrm{R}$ 2.8.0, $R$ Foundation for Statistical Computing, Vienna, Austria).

Relationships between response variables and climate. We investigated the relationships between the magnitudes of responses of plant growth and ecosystem $\mathrm{C}$ balance to climate change treatments and climate, using the sensitivity metric because it standardizes treatment effects to the magnitude of the treatment imposed, yielding effect sizes normalized for treatments across all ecosystems. We used linear regression to investigate the relationships between effect sizes and MAT and MAP in $\mathrm{R}$ ( $\mathrm{R}$ 2.8.0, $R$ Foundation for Statistical Computing, Vienna, Austria).

\section{Results}

\section{Effects of climate change treatments on plant biomass}

Warming enhanced aboveground biomass across the experiments surveyed, but did not significantly affect total or belowground biomass (Table 3). Warming significantly stimulated total biomass when weighted by duration of the experiment (Table 3), indicating that positive responses became more pronounced in long- term experiments. Warming increased aboveground biomass on average by $27 \%\left(41.9,18.6-72.0 \mathrm{~g} \mathrm{~m}^{-2}\right)$. Total biomass and aboveground biomass showed significantly positive sensitivities of $242.4(12.4-447.8) \mathrm{g} \mathrm{m}^{-2}{ }^{\circ} \mathrm{C}^{-1}$ and 50.5 (17.7-112.3) $\mathrm{g} \mathrm{m}^{-2}{ }^{\circ} \mathrm{C}^{-1}$ to warming, respectively. The high values reflect large changes in total and aboveground biomass in response to $<1{ }^{\circ} \mathrm{C}$ change in soil temperature, which occurred in a number of cases (Wookey et al., 1995; Press et al., 1998; Jonasson et al., 1999; Saleska et al., 2002; Kudo \& Suzuki, 2003; Dukes et al., 2005; Jónsdóttir et al., 2005; Biasi et al., 2008; Sardans et al., 2008). Decreased precipitation suppressed aboveground biomass, whereas increased precipitation stimulated aboveground and belowground biomass (Table 3). Reduced precipitation suppressed aboveground biomass by $15 \%\left(19.6,3.6-49.3 \mathrm{~g} \mathrm{~m}^{-2}\right)$. Increased precipitation stimulated aboveground biomass on average by $12 \%$ $\left(12.1,-1.7\right.$ to $\left.27.8 \mathrm{~g} \mathrm{~m}^{-2}\right)$ and belowground biomass by $11 \%\left(20.3,7.5-45.0 \mathrm{~g} \mathrm{~m}^{-2}\right)$. Both aboveground and belowground biomass showed positive sensitivities to increased precipitation, and aboveground biomass showed positive sensitivity to decreased precipitation as well (Table 4). Aboveground biomass showed no difference in sensitivity to increased and reduced precipitation treatments (Table 4). There was no evidence for variation of responses of plant biomass to warming or altered precipitation as a function of climate (Table 5).

The combined effects of experimental warming and altered precipitation on plant biomass and productivity

Table 3 Log response ratio effect size metrics of biomass, net primary production (NPP), respiration, net ecosystem exchange and ecosystem photosynthesis under temperature and precipitation manipulation

\begin{tabular}{|c|c|c|c|}
\hline & Warming & Decreased precipitation & Increased precipitation \\
\hline Total biomass & $\begin{array}{lll}0 & 0 & 0\end{array}+(7)$ & na & na \\
\hline Aboveground biomass & $++++(32)$ & $----(10)$ & $++++(19)$ \\
\hline Belowground biomass & $\begin{array}{llll}-0 & 0 & 0 & (6)\end{array}$ & na & $++++(4)$ \\
\hline TNPP & $++++(6)$ & na & $++++(2)$ \\
\hline ANPP & $\begin{array}{lllll}0 & 0 & 0 & 0 & (18)\end{array}$ & $----(14)$ & $++++(14)$ \\
\hline BNPP & $++++(5)$ & na & $++++(4)$ \\
\hline Ecosystem respiration & $++++(28)$ & $0000(4)$ & $++++(16)$ \\
\hline Aboveground respiration & $++++(2)$ & na & $\begin{array}{lllll}0 & 0 & 0 & 0 & (5)\end{array}$ \\
\hline Soil respiration & $+0++(27)$ & $----(8)$ & $+0++(16)$ \\
\hline Net ecosystem exchange* & $\begin{array}{lllll}0 & 0 & 0 & 0 & (26)\end{array}$ & $----(4)$ & $++++(16)$ \\
\hline Ecosystem photosynthesis & $++++(24)$ & $----(4)$ & $++++(17)$ \\
\hline
\end{tabular}

Treatments include warming, decreased precipitation and increased precipitation. Significance of effect size is shown by $(+) 95 \%$ bootstrapped confidence interval is greater than zero; (0) 95\% bootstrapped confidence interval overlapped zero; (-) 95\% bootstrapped confidence interval is smaller than zero. Multiple symbols indicate respectively the significance of effect size calculated using equal weights, weighting by inverse of pooled variance, weighting by sample size, and weighting by experimental duration. The number of experiments included in the analysis for the response variables under climate change treatments are in parentheses.

*Positive effect sizes indicate increases in net $\mathrm{C}$ uptake, and negative effect size indicate decreases in net $\mathrm{C}$ uptake. na, not available (sample size is not sufficient for the analysis); TNPP, total NPP; ANPP, Aboveground NPP; BNPP, Belowground NPP. 
Table 4 Sensitivity of response variables to experimentally altered precipitation weighting by sample size

\begin{tabular}{|c|c|c|c|c|}
\hline \multirow[b]{2}{*}{ Variables } & \multicolumn{2}{|c|}{ Decreased precipitation } & \multicolumn{2}{|c|}{ Increased precipitation } \\
\hline & Mean & 95\% Bootstrapped CI & Mean & $95 \%$ Bootstrapped CI \\
\hline Total biomass $\left(\mathrm{g} \mathrm{m}^{-2} \mathrm{~mm}^{-1}\right)$ & - & - & - & - \\
\hline Aboveground biomass $\left(\mathrm{g} \mathrm{m}^{-2} \mathrm{~mm}^{-1}\right)$ & 0.16 & $0.04-0.33$ & 0.30 & $0.03-0.66$ \\
\hline Belowground biomass $\left(\mathrm{g} \mathrm{m}^{-2} \mathrm{~mm}^{-1}\right)$ & - & - & 0.09 & $0.03-0.20$ \\
\hline Total NPP (TNPP, $\mathrm{g} \mathrm{m}^{-2} \mathrm{yr}^{-1} \mathrm{~mm}^{-1}$ ) & - & - & 0.04 & $0.01-0.14$ \\
\hline Aboveground NPP (ANPP, $\mathrm{g} \mathrm{m}^{-2} \mathrm{yr}^{-1} \mathrm{~mm}^{-1}$ ) & 0.19 & $0.08-0.32$ & 0.67 & $0.34-1.16$ \\
\hline Belowground NPP (BNPP, $\mathrm{g} \mathrm{m}^{-2} \mathrm{yr}^{-1} \mathrm{~mm}^{-1}$ ) & - & - & 0.12 & $0.02-0.28$ \\
\hline Ecosystem respiration $\left(\mu \mathrm{molCO}_{2} \mathrm{~m}^{-2} \mathrm{~s}^{-1} \mathrm{~mm}^{-1}\right)$ & 0.001 & $0.0007-0.002$ & 0.02 & $0.007-0.03$ \\
\hline Aboveground respiration $\left(\mu \mathrm{mol} \mathrm{CO} \mathrm{m}^{-2} \mathrm{~s}^{-1} \mathrm{~mm}^{-1}\right)$ & - & - & -0.001 & -0.003 to 0.002 \\
\hline Soil respiration $\left(\mu \mathrm{molCO} \mathrm{Cm}^{-2} \mathrm{~s}^{-1} \mathrm{~mm}^{-1}\right)$ & 0.0005 & $0.0001-0.001$ & 0.02 & $0.006-0.05$ \\
\hline Net ecosystem exchange $\left(\mu \mathrm{molCO} \mathrm{Cm}^{-2} \mathrm{~s}^{-1} \mathrm{~mm}^{-1}\right)$ & 0.001 & $0.0007-0.002$ & 0.06 & $0.01-0.14$ \\
\hline Ecosystem photosynthesis $\left(\mu \mathrm{mol} \mathrm{CO} \mathrm{Cm}^{-2} \mathrm{~s}^{-1} \mathrm{~mm}^{-1}\right)$ & 0.001 & $0.0005-0.002$ & 0.03 & $0.01-0.06$ \\
\hline
\end{tabular}

were not simply predicted by their effects measured in isolation (Fig. 2). Observed responses tended to be smaller in absolute value than the expected responses based on the additive combination of single factor effects (slope significantly $<1, P=0.02$ ). When singlefactor effect sizes were small, interactions were not apparent (intercept not significantly different from 0 , $P=0.10$ ). Thus, interactions between warming and altered precipitation, when they occurred, tended to be more muted than single-factor experiments might suggest.

\section{Effects of climate change treatments on plant productivity}

Warming enhanced TNPP and BNPP across all study sites, but showed no significant effects on ANPP (Table 3). TNPP was stimulated by warming by an average of $15 \%\left(58.9,35.2-95.7 \mathrm{~g} \mathrm{~m}^{-2} \mathrm{yr}^{-1}\right)$ and BNPP by $52 \%(58.4$, 51.3-66.6 $\mathrm{g} \mathrm{m}^{-2} \mathrm{yr}^{-1}$ ). TNPP and BNPP showed sensitivities to warming of $38.9(26.5-50.5) \mathrm{g} \mathrm{m}^{-2} \mathrm{yr}^{-1}{ }^{\circ} \mathrm{C}^{-1}$ and 26.7 (20.1-33.8) $\mathrm{g} \mathrm{m}^{-2} \mathrm{yr}^{-1}{ }^{\circ} \mathrm{C}^{-1}$, respectively. Decreased precipitation significantly reduced ANPP, whereas increased precipitation stimulated total, aboveground, and belowground plant productivity (Table 3 ). ANPP was suppressed by decreased precipitation on average by $37 \%\left(33.7,13.8-60.5 \mathrm{~g} \mathrm{~m}^{-2} \mathrm{yr}^{-1}\right)$, with a significantly positive sensitivity (Table 4 ). Increased precipitation stimulated ANPP by $28 \%$ (103.7, 44.0$194.6 \mathrm{~g} \mathrm{~m}^{-2} \mathrm{yr}^{-1}$ ), exhibiting stronger sensitivity to increased than to reduced precipitation (Table 4). Supplemental precipitation also stimulated TNPP by $4 \%$ (16.1, $\left.0.1-59.0 \mathrm{~g} \mathrm{~m}^{-2} \mathrm{yr}^{-1}\right)$, and BNPP by $6 \%$ (86.0, 12.3$157.3 \mathrm{~g} \mathrm{~m}^{-2} \mathrm{yr}^{-1}$ ). TNPP, ANPP, and BNPP all showed significantly positive sensitivities to increased precipitation (Table 4).

Responses of ANPP to warming decreased with MAP $(P=0.001$, Table 5$)$, suggesting dry ecosystem were more responsive to warming; responses of ANPP to increased precipitation declined with MAT $(P=0.003$, Table 5), indicating cold ecosystems were more responsive to supplemental precipitation. No other significant relationships were observed between effect sizes of plant productivity with climate (Table 5).

Combined effects of warming and altered precipitation on ANPP were similar to those observed for aboveground biomass (Fig. 2). The slope of the linear regression was significantly $<1(P=0.04)$ and intercept not significantly different from $0(P=0.99)$, indicating that the observed responses of ANPP to the combined effects of warming and altered precipitation effects were smaller than expected based on single-factor manipulations.

\section{Effects of climate change treatments on respiration}

Warming increased ecosystem respiration, aboveground respiration, and soil respiration (Table 3). Decreased precipitation suppressed soil respiration, but did not significantly alter ecosystem respiration (Table 3). Increased precipitation enhanced ecosystem and soil respiration, but had no significant effects on aboveground respiration (Table 3). Warming increased ecosystem respiration on average by $27 \%(0.32,0.06-$ $0.58 \mu \mathrm{mol} \mathrm{CO} \mathrm{CO}_{2} \mathrm{~m}^{-2} \mathrm{~s}^{-1}$ ), aboveground respiration by $15 \%\left(0.52,0.33-0.62 \mu \mathrm{mol} \mathrm{CO} \mathrm{Cm}^{-2} \mathrm{~s}^{-1}\right)$, and soil respiration by $12 \%\left(0.37,0.13-0.64 \mu \mathrm{mol} \mathrm{CO} \mathrm{Cm}^{-2} \mathrm{~s}^{-1}\right)$, respectively. In addition, aboveground respiration showed a significantly positive sensitivity to warming of 0.30 (0.13-0.39) $\mu \mathrm{mol} \mathrm{CO} 2 \mathrm{~m}^{-2} \mathrm{~s}^{-1}{ }^{\circ} \mathrm{C}^{-1}$. Decreased precipitation reduced soil respiration by $12 \%(0.28,0.02-$ $0.74 \mu \mathrm{mol} \mathrm{CO} \mathrm{CO}^{-2} \mathrm{~s}^{-1}$ ), whereas increased precipitation stimulated ecosystem respiration on average by $30 \%$ $\left(0.57,0.23-0.98 \mu \mathrm{mol} \mathrm{CO}_{2} \mathrm{~m}^{-2} \mathrm{~s}^{-1}\right)$ and soil respiration by $45 \%\left(1.36,0.50-2.58 \mu \mathrm{mol} \mathrm{CO} \mathrm{m}^{-2} \mathrm{~s}^{-1}\right)$. Both ecosys- 
Table 5 Linear regression analyses for relationships between sensitivity effect sizes (weighting by sample size) and climate including mean annual temperature (MAT) and mean annual precipitation (MAP)

\begin{tabular}{|c|c|c|c|c|c|c|c|c|c|}
\hline \multirow[b]{2}{*}{ Variables } & \multirow{2}{*}{$\begin{array}{l}\text { Climate change } \\
\text { treatment }\end{array}$} & \multicolumn{4}{|l|}{ MAT } & \multicolumn{4}{|l|}{ MAP } \\
\hline & & Intercept & Slope & $R^{2}$ & $P$-value & Intercept & Slope & $R^{2}$ & $P$-value \\
\hline Total biomass & $\mathrm{W}$ & 252.2 & 2.7 & 4.E-03 & ns & 423.3 & -0.4 & 0.2 & ns \\
\hline \multirow[t]{3}{*}{ Aboveground biomass } & $\mathrm{W}$ & 82.6 & -6.3 & 7.E-02 & ns & 129.4 & -0.1 & 0.1 & ns \\
\hline & DP & 0.2 & $-4 . \mathrm{E}-03$ & 1.E-02 & ns & -0.1 & 1.E-03 & 0.7 & ns \\
\hline & $\mathrm{IP}$ & 0.5 & $-1 . \mathrm{E}-02$ & 1.E-02 & ns & 0.5 & $-2 . \mathrm{E}-04$ & 1.E-02 & ns \\
\hline \multirow[t]{2}{*}{ Belowground biomass } & $\mathrm{W}$ & -13.5 & 1.3 & 0.2 & ns & 2.0 & $-1 . E-02$ & 2.E-02 & ns \\
\hline & IP & 0.1 & 3.E-04 & 4.E-04 & ns & 0.1 & 2.E-05 & 1.E-03 & ns \\
\hline Total NPP (TNPP) & $\mathrm{W}$ & 34.9 & 0.4 & 1.E-02 & ns & 28.5 & 1.E-02 & 1.E-02 & ns \\
\hline \multirow[t]{3}{*}{ Aboveground NPP (ANPP) } & $\mathrm{W}$ & -20.9 & 1.0 & 1.E-02 & ns & 98.2 & -0.2 & 0.6 & $0.001^{*}$ \\
\hline & $\mathrm{DP}$ & 0.7 & $-4 . \mathrm{E}-02$ & 0.2 & ns & $-4 . \mathrm{E}-02$ & 4.E-04 & 0.4 & ns \\
\hline & $\mathrm{IP}$ & 2.0 & -0.1 & 0.3 & $0.003^{*}$ & 1.1 & $-3 . \mathrm{E}-04$ & 1.E-02 & ns \\
\hline Belowground NPP (BNPP) & W & 25.6 & 0.1 & 1.E-02 & ns & 22.9 & 1.E-02 & 1.E-02 & ns \\
\hline \multirow[t]{3}{*}{ Ecosystem respiration } & W & 0.2 & $-1 . \mathrm{E}-02$ & 3.E-02 & ns & 0.2 & 7.E-05 & 2.E-03 & ns \\
\hline & $\mathrm{DP}$ & 6.E-04 & 5.E-05 & 7.E-02 & ns & 2.E-03 & $-1 . \mathrm{E}-06$ & 0.1 & ns \\
\hline & IP & 3.E-02 & $-2 . \mathrm{E}-03$ & 5.E-02 & ns & 1.E-02 & 3.E-05 & 1.E-02 & ns \\
\hline Aboveground respiration & $\mathrm{IP}$ & $-2 . \mathrm{E}-03$ & 4.E-04 & 0.1 & ns & 1.E-02 & $-2 . E-05$ & 0.1 & ns \\
\hline \multirow[t]{3}{*}{ Soil respiration } & $\mathrm{W}$ & 0.1 & $-4 . \mathrm{E}-03$ & 1.E-02 & ns & 0.2 & $-1 . \mathrm{E}-04$ & 2.E-02 & ns \\
\hline & DP & 1.E-03 & $-4 . \mathrm{E}-06$ & 1.E-03 & ns & 1.E-03 & $-4 . \mathrm{E}-07$ & 6.E-02 & ns \\
\hline & IP & 4.E-02 & $-2 . \mathrm{E}-03$ & 0.2 & ns & 8.E-02 & $-1 . \mathrm{E}-04$ & 0.2 & $0.03^{*}$ \\
\hline \multirow[t]{3}{*}{ Net ecosystem exchange (NEE) } & $\mathrm{W}$ & -0.2 & 2.E-02 & 0.1 & ns & 0.1 & -0.001 & 0.1 & ns \\
\hline & $\mathrm{DP}$ & $-1 . \mathrm{E}-03$ & 2.E-04 & 0.4 & ns & 2.E-03 & $-4 . E-06$ & 0.4 & ns \\
\hline & IP & 3.E-02 & $-2 . \mathrm{E}-03$ & 0.1 & ns & $-5 . \mathrm{E}-03$ & 7.E-05 & 3.E-02 & ns \\
\hline \multirow[t]{3}{*}{ Ecosystem photosynthesis } & $\mathrm{W}$ & 0.2 & $-1 . \mathrm{E}-02$ & 2.E-02 & ns & $-4 . \mathrm{E}-02$ & 1.E-03 & 0.1 & ns \\
\hline & $\mathrm{DP}$ & 3.E-03 & $-2 . \mathrm{E}-04$ & 0.3 & ns & 3.E-04 & 3.E-06 & 0.2 & ns \\
\hline & IP & 0.1 & $-4 . \mathrm{E}-03$ & 0.1 & ns & 5.E-02 & $-2 . \mathrm{E}-05$ & 2.E-03 & ns \\
\hline
\end{tabular}

*Significant $(P<0.05)$.

$\mathrm{W}$, warming; DP, decreased precipitation; IP, increased precipitation; ns, nonsignificant.

tem and soil respiration showed significantly higher sensitivities to increased precipitation than to decreased precipitation (Table 4). Respiration in ecosystems dominated by herbaceous and woody vegetation responded equally to temperature and precipitation treatments.

The relative effects of increased precipitation on soil respiration declined with increasing MAP $(P=0.03$, Table 5), suggesting that increased precipitation has a larger stimulating effect on soil respiration in dry ecosystems. No other significant relationship between effects of warming and altered precipitation on respiration and climate was observed (Table 5).

The combined effects of warming and altered precipitation on ecosystem respiration were smaller than expected based on combined additive responses, indicated by a slope of the linear regression significantly $<1 \quad(P=0.03$, Fig. 3$)$. When single-factor effect sizes were small, a lack of interactive effects were suggested by an intercept not significantly different from 0 $(P=0.75)$.

\section{Effects of climate change treatments on NEE}

Warming showed no significant effects on NEE. Decreased precipitation suppressed NEE, while increased precipitation stimulated NEE (Table 3). Decreased precipitation reduced NEE on average by $45 \%(0.09,0.06-$ $0.13 \mu \mathrm{molCO}_{2} \mathrm{~m}^{-2} \mathrm{~s}^{-1}$ ) and increased precipitation stimulated NEE by $56 \%\left(0.40,0.01-0.89 \mu \mathrm{molCO}_{2} \mathrm{~m}^{-2}\right.$ $\mathrm{s}^{-1}$ ). NEE was significantly more sensitive to increased precipitation than to decreased precipitation (Table 4). Similar to plant biomass and productivity, the effects of warming on net $C$ uptake were similar for ecosystems dominated by herbaceous and woody vegetation. The response of NEE to warming and altered precipitation did not vary with climate (Table 5). The slope of interactive against additive effects of warming and altered precipitation did not differ significantly from 1 $(P=0.68$, Fig. 3$)$, and the intercept did not differ from $0(P=0.10)$. Therefore, responses of NEE to temperature and precipitation manipulations in isolation were sufficient to predict their combined effects. 


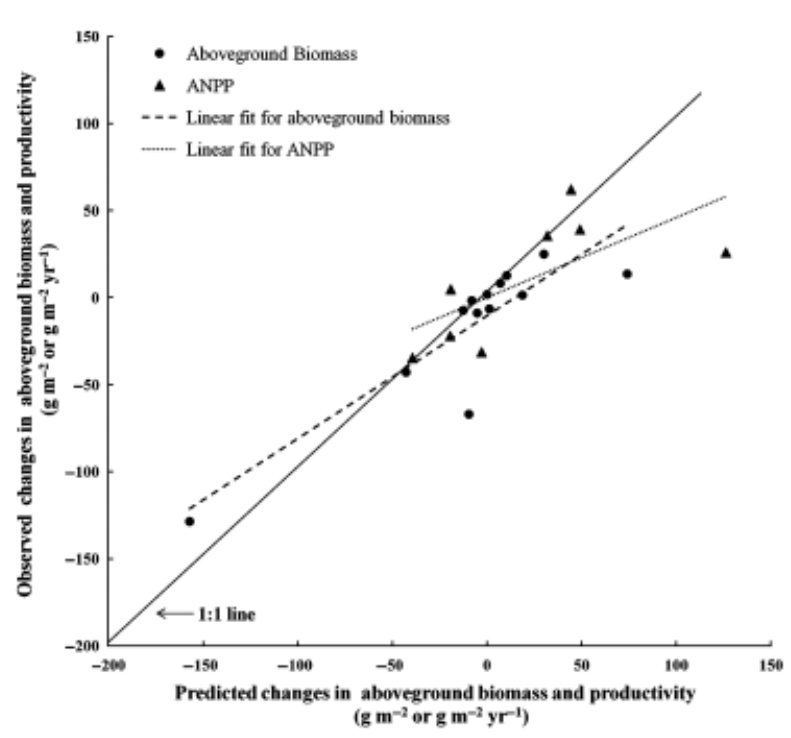

Fig. 2 Interactive effects of temperature and precipitation treatments on aboveground biomass $\left(\mathrm{g} \mathrm{m}^{-2}\right)$ and aboveground net primary productivity (ANPP, $\mathrm{g} \mathrm{m}^{-2} \mathrm{yr}^{-1}$ ). Predicted values are the sums of absolute effect sizes from single-factor manipulation, i.e., assuming effects of altered precipitation and warming are additive. The solid line is the $1: 1$ line, expected if interactions are absent. Linear regression for aboveground biomass (dashed line) is $y=-10.29+0.71 x(P<0.001)$; the slope is significantly $<1(P=0.02)$ and the intercept is not significantly different from $0 \quad(P=0.10)$. Linear regression for ANPP (dotted line) is $y=0.14+0.46 x \quad(P=0.07) ;$ the slope is significantly $<1$ $(P=0.04)$ and the intercept is not significantly different from 0 $(P=0.99)$.

\section{Effects of climate change treatments on ecosystem photosynthesis}

Warming and increased precipitation stimulated ecosystem photosynthesis, and decreased precipitation suppressed ecosystem photosynthesis (Table 3). Warming enhanced ecosystem photosynthesis on average by $20 \%\left(0.19,-0.05\right.$ to $\left.0.47 \mu \mathrm{molCO}_{2} \mathrm{~m}^{-2} \mathrm{~s}^{-1}\right)$ and increased precipitation stimulated ecosystem photosynthesis by $40 \%\left(1.13,0.52-1.89 \mu \mathrm{mol} \mathrm{CO}_{2} \mathrm{~m}^{-2} \mathrm{~s}^{-1}\right)$. Ecosystem photosynthesis was reduced by decreased precipitation by $9 \%\left(0.12,0.03-0.26 \mu \mathrm{molCO}_{2} \mathrm{~m}^{-2} \mathrm{~s}^{-1}\right)$. Ecosystem photosynthesis of herbaceous and woody vegetation responded similarly to experimental warming. Ecosystem photosynthesis showed a significantly positive sensitivity of $0.22(0.04-0.42) \mu \mathrm{mol} \mathrm{CO}_{2} \mathrm{~m}^{-2} \mathrm{~s}^{-1}$ ${ }^{\circ} \mathrm{C}^{-1}$ to warming, and a higher sensitivity to increased than decreased precipitation (Table 4). No significant relationship was observed between effects of experimental warming and altered precipitation on ecosystem photosynthesis and climate among different ecosystems (Table 5). Additive combinations of the responses of

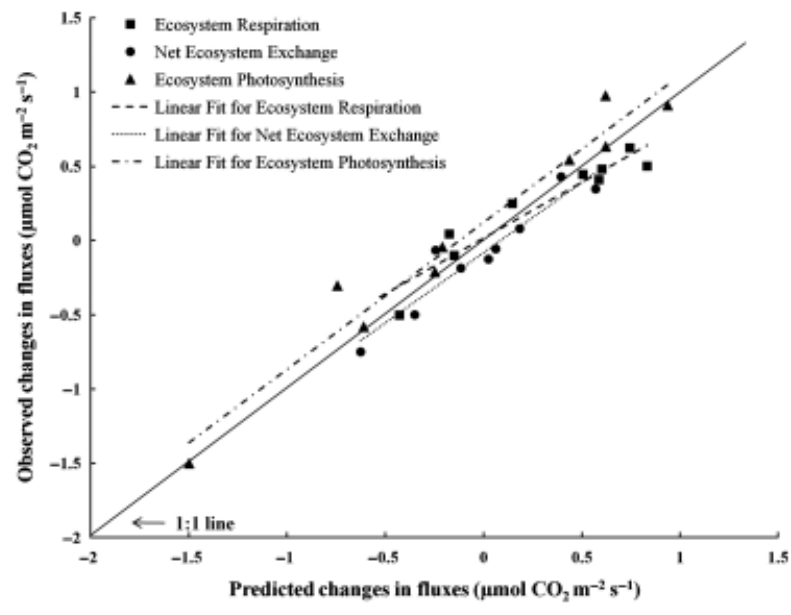

Fig. 3 Interactive effects of temperature and precipitation treatments on ecosystem respiration, ecosystem photosynthesis and net ecosystem exchange (NEE, $\mu \mathrm{mol} \mathrm{CO}_{2} \mathrm{~m}^{-2} \mathrm{~s}^{-1}$ ). Predicted values are the sums of absolute effect sizes from single-factor manipulation, i.e., assuming effects of altered precipitation and warming are additive. The solid line is the $1: 1$ line, expected if interactions are absent. Linear regression for ecosystem respiration (dashed line) is $y=0.02+0.75 x(P<0.001)$; the slope is significantly $<1(P=0.03)$ and the intercept is not significantly different from $0(P=0.75)$. Linear regression for NEE (dotted line $)$ is $y=-0.08+0.95 x(P<0.001)$; the slope is not significantly different from $1(P=0.68)$ and intercept is not significantly different from $0(P=0.10)$. Linear regression for ecosystem photosynthesis (dash-dotted line) is $y=0.12+0.99 x(P<0.001)$; the slope is not significantly different from $1(P=0.89)$ and the intercept is not significantly different from $0(P=0.08)$.

ecosystem photosynthesis to temperature and precipitation predicted well their combined effects (Fig. 3), as indicated by a slope not significantly different from 1 $(P=0.89)$ and an intercept not significantly different from $0(P=0.08)$.

\section{Discussion}

\section{Effects of elevated temperature}

We found that warming increased both respiration and ecosystem photosynthesis, but showed no significant effects on net $\mathrm{C}$ uptake. Meanwhile, warming also generally increased plant biomass and productivity. Another meta-analysis of ecosystem warming experiments (Rustad et al., 2001) concluded that warming increased soil respiration and plant productivity, with a larger response in woody ecosystems. Our larger data set generally showed no difference in sensitivity to warming between ecosystems dominated by herbaceous and woody vegetation. Dormann \& Woodin 
(2002) reviewed 36 experiments from the arctic and also found that warming increased biomass. Warming stimulated plant productivity (Peñuelas et al., 2007; Luo et al., 2009), and also enhanced ecosystem photosynthesis because of increasing aboveground biomass (Sullivan et al., 2008), due to enhanced soil nutrient mineralization (Hartley et al., 1999; Grogan \& Chapin, 2000; Melillo et al., 2002), compensating for increased respiratory C losses (Melillo et al., 2002). In one case, warming increased soil organic C content (Sardans et al., 2008), perhaps because of suppressed microbial activity as a result of soil drying, as found in a mature black spruce forest (Allison \& Treseder, 2008). Other studies have shown that experimental warming decreased plant biomass and photosynthesis due to warming-induced moisture stress (De Valpine \& Harte, 2001; De Boeck et al., 2007, 2008), suppressing both ecosystem photosynthesis and soil respiration, with the latter declining more (Liu et al., 2009). In most experiments, warming increased soil respiration (Mertens et al., 2001; Emmett et al., 2004; Biasi et al., 2008), due to higher activity of microbes and roots (Bergner et al., 2004; Sardans et al., 2008), and increased C input from plant production (Welker et al., 2004; Luo et al., 2009). Increasing respiratory $C$ losses could transform a $\mathrm{C}$ sink into a $\mathrm{C}$ source, as responses of respiration can dominate effects on ecosystem net $\mathrm{C}$ balance (Illeris et al., 2004; Oberbauer et al., 2007). However, warming-induced respiratory $\mathrm{CO}_{2}$ losses could decline because of lower litter quality from species composition shifts (Harte \& Shaw, 1995; Biasi et al., 2008).

Overall, we found little influence of experimental duration on the significance of results, except that warming-induced increases in total biomass only appeared in long-term experiments. Most studies included in this analysis were short term ( $<5$ years), and warming-induced vegetative growth was not limited by nutrients due to increased litter decomposition and nutrient availability. Warming increased nitrogen (N) mineralization (Hartley et al., 1999; Melillo et al., 2002), and redistribution of $\mathrm{N}$ from soil to plants should eventually reach a point where labile $\mathrm{N}$ pools in soil cannot support increased aboveground growth, and respiratory $C$ losses will dominate the overall $C$ balance (Shaver et al., 2000). For example, warming did not show any effect in the 5th year of treatment on a subarctic dwarf shrub heath (Hartley et al., 1999). Arft et al. (1999) also found enhanced vegetative growth for the first 3 years, but no significant response for the fourth year. Initial increase of soil respiration is from the consumption of soil labile C (McHale et al., 1998), and such responses can be transient because of the depletion of the labile soil C pool (Melillo et al., 2002). Long-term soil warming can also cause microbial acclimation
(Zogg et al., 1997) and root acclimation (Atkin et al., 2000), resulting in little or no response of ecosystem respiration to warming. Long-term warming could also induce soil drying that will suppress soil respiration (McHale et al., 1998). Such long-term responses may be difficult to capture in warming experiments, yet it is important to distinguish transient patterns from longterm responses.

Rustad et al. (2001) used meta-analysis of warming experiments and found larger responses of plant productivity to warming in colder environments. However, no trend in our dataset was observed between responses of plant productivity to experimental warming and MAT. This difference between the two meta-analyses could result from sample size - our analysis included more studies covering a broader geographical range than was available to Rustad et al. (2001). Soil respiration has been long recognized as being temperature-dependent, often modeled with a $Q_{10}$ function (Schleser, 1982; Chen \& Tian, 2005). However, some lines of evidence suggest that sensitivity of soil respiration acclimates in response to warming (Luo et al., 2001), such that increases in soil respiration are smaller than expected from the $Q_{10}$ function. Kirschbaum (1995) found that soil respiration had higher sensitivity in colder environments, but this pattern was not apparent in our analysis.

Global mean temperature is predicted to increase $2-7^{\circ} \mathrm{C}$ by the end of this century (Allison et al., 2009). The sensitivity of ecosystem photosynthesis we found $\left(0.22 \mu \mathrm{mol} \mathrm{CO}_{2} \mathrm{~m}^{-2} \mathrm{~s}^{-1}{ }^{\circ} \mathrm{C}^{-1}\right)$ in response to warming is nearly exactly balanced by sensitivity of ecosystem respiration $\left(0.21 \mu \mathrm{molCO}_{2} \mathrm{~m}^{-2} \mathrm{~s}^{-1}{ }^{\circ} \mathrm{C}^{-1}\right)$, suggesting that short-term responses of both processes to projected warming are on the order of $0.4-1.5 \mu \mathrm{mol} \mathrm{CO} \mathrm{CO}^{-2} \mathrm{~s}^{-1}$ (depending on the magnitude of the temperature increase). Whether these effects cancel or shift toward net carbon uptake or release will depend on processes that operate on longer time scales than accessible in manipulative experiments.

\section{Effects of altered precipitation}

We found that increased precipitation stimulated both respiration and ecosystem photosynthesis, and led to an overall increase in net $C$ uptake, reflected in both increased plant biomass and productivity. Chen et al. (2009) showed that gross ecosystem productivity had a higher sensitivity to soil moisture than that of soil respiration, and therefore increased precipitation favors C sequestration. Our results contrast with a prior assessment of arctic experiments, which showed no significant effects of increased precipitation on plant biomass (Dormann \& Woodin, 2002). We also synthe- 
sized effects of decreased precipitation on ecosystem C balance and found that reduced precipitation suppressed both soil respiration and ecosystem photosynthesis, and resulted in an overall decrease in net $\mathrm{C}$ uptake, also reflected in decreased aboveground biomass and productivity. Decreased precipitation can also reduce nutrient availability because of water limitation of soil microbial processes (De Dato et al., 2006; Sardans et al., 2008). Decreased precipitation not only suppresses plant biomass and physiological processes, it can also cause mortality, as shown in a holm oak forest (Ogaya \& Peñuelas, 2007). Ecosystem $C$ cycling responded to both increased and decreased precipitation, with higher sensitivities to supplemental precipitation than to reduced precipitation. We also found ANPP had a significantly higher sensitivity to increased precipitation than that to decreased precipitation, similar to the prediction from long-term relationships between ANPP and annual precipitation (Knapp \& Smith, 2001).

Our results showed the effects of altered total precipitation quantity on plant growth and ecosystem C fluxes, yet the timing and frequency of precipitation can also have large effects (Knapp et al., 2008). Extension of the wet season increased microbial respiration and $\mathrm{C}$ uptake from enhanced plant productivity (Silver $e t$ al., 2005). Alteration of precipitation timing in a sagebrush steppe caused plant productivity change and vegetation shifts (Bates et al., 2006). Rainfall timing, such as the interval between rainfall events, influenced the productivity of grassland ecosystems (Fay et al., 2000). Soil $\mathrm{CO}_{2}$ flux declined more because of altered rainfall timing than reduced rainfall amount, with the combination of both causing the largest reduction (Harper et al., 2005). Rain pulse sizes also affected soil respiration (Chen et al., 2008), C sequestration and plant productivity (Heisler-White et al., 2008; Chen et al., 2009). Thus, the inferences from our results could be modified if changes in precipitation timing and frequency are considered.

The effect of moisture on soil respiration is complex, not well explained by simple linear relationships (Howard \& Howard, 1979; Davidson et al., 1998). Our finding that soil respiration was more responsive to increased precipitation in dry environments suggests that relative water limitation of these processes declines with increasing water availability. The global precipitation trends ranged from -7 to $+2 \mathrm{~mm}$ per decade according to the IPCC (Solomon et al., 2007). Based on our analysis of sensitivity to precipitation change, shortterm responses of NEE to observed precipitation trends might range from a decrease of $0.007 \mu \mathrm{molCO}_{2} \mathrm{~m}^{-2} \mathrm{~s}^{-1}$ to an increase of $0.12 \mu \mathrm{molCO}_{2} \mathrm{~m}^{-2} \mathrm{~s}^{-1}$ (excluding influences of longer-term responses to precipitation, such as land-use changes and species composition shifts).

\section{Temperature and precipitation interactions}

Based on a small number of experiments that manipulated both temperature and precipitation, we found that ecosystem responses to the combination of warming and altered precipitation tended to be smaller than expected from the single-factor responses. Luo et al. (2008) modeled interactive effects of precipitation and temperature on ecosystem $C$ dynamics, and showed that warming and doubled precipitation generally have positive effects on NPP, net ecosystem production, and respiration, whereas warming and reduced (halved) precipitation have negative effects on NPP. Similar to our results, the modeling study showed interactive effects were generally small (Luo et al., 2008).

\section{Conclusions}

Meta-analysis supported some general conclusions about ecosystem responses to climate change: (1) Warming increased plant biomass and productivity, respiration and ecosystem photosynthesis, but did not affect net $C$ uptake. (2) Increased precipitation stimulated plant biomass, productivity, respiration, ecosystem photosynthesis, and net $C$ uptake. (3) Decreased precipitation suppressed aboveground biomass and productivity, soil respiration, ecosystem photosynthesis, and net $C$ uptake. (4) Plant productivity and ecosystem $\mathrm{C}$ fluxes were more sensitive to increased precipitation than to reduced precipitation. (5) Herbaceous and woody plants showed similar responses to climate change treatments. (6) When interactions occurred between warming and altered precipitation, the combined responses tended to be smaller than expected from additive, single-factor effects. Finally, (7) the magnitude of responses of these ecosystem processes exhibited little systematic variation with climate, indicating general sensitivities across ecosystems to climate change treatments.

For future experiments on ecosystem-level responses to climate change treatments, we recommend:

(1) Measure total and belowground biomass and productivity in addition to aboveground biomass and productivity. Aboveground biomass and productivity are commonly used to estimate responses of plant growth to climate change. However, belowground biomass and productivity play an important role in such responses, with which total biomass and productivity can be calculated to quantify ecosystem level responses to climate change.

(2) Conduct more experiments manipulating precipitation. Because of the variability and unpredictability of future precipitation projections, more precipitation manipulation experiments are needed to eluci- 
date the impacts of wide range of possible scenarios. These experiments should manipulate not only precipitation quantity, but also alter precipitation timing, frequency, intensity as well as seasonality.

(3) Design multifactorial experiments in a wide range of ecosystems. Temperature and precipitation effects could be additive, so single-factor experiments can be very informative and provide the basic mechanisms for ecosystem responses. However, complex interactions do exist and may not be consistent among ecosystems or treatments. In this sense, a single factor experiment is not adequate to illustrate the responses of ecosystem under interactive climate change effects.

(4) Establish experiments in underrepresented biomes and environments. Multiple-factor experiments have been limited to herbaceous ecosystems. Yet, given the greater biomass, soil microbial biomass, soil $\mathrm{C}$ pools, and high $\mathrm{C}$ fluxes in woody communities, it is crucial to include more woody systems in multifactor manipulation experiments. However, the technological and cost constrains make mature forest ecosystem warming experiments very difficult. In addition, most manipulation experiments have been in mid-to-high latitudes in northern hemisphere, and new experiments are needed in low latitude and tropical systems to identify a systematic variation of responses across ecosystems.

\section{Acknowledgements}

Thanks to Gaius Shaver, Rebecca Sherry, Albert Tietema, Malcolm Press, Ken Thompson, Phillips Donald, Claus Beier, Iain Hartley, Steven Link, Evan Siemann, Hans De Boeck, Angela Breeuwer, Bjorn Robroek, Rich Conant, Steve Oberbauer, Jake Weltzin, Jim Lewis, Scott Bridgham, Ton Gorissen, Juan de Dios Miranda, Mike Laporte, Shiqiang Wan, Juergen Kreyling, Julianne Lilley, David Olszyk, and Anita Risch for sharing their data. This work was supported by the National Institute of Climatic Change Research, the US National Science Foundation (DEB-0092642), the government of Catalunya (AGAUR 2005PIV00123, Grant SGR2009-458), the Spanish Government (Grants CGL200604025/BOS and Consolider Ingenio Montes CSD2008-00040), and Science Foundation Arizona (GRF 0001-07).

\section{References}

Allison I, Bindoff NL, Bindoff RA et al. (2009) The Copenhagen Diagnosis, 2009: Updating the World on the Latest Climate Science. The University of New South Wales Climate Change Research Centre (CCRC), Sydney, Australia.

Allison SD, Treseder KK (2008) Warming and drying suppress microbial activity and carbon cycling in boreal forest soils. Global Change Biology, 14, 2898-2909.

Angert A, Biraud S, Bonfils C et al. (2005) Drier summers cancel out the $\mathrm{CO}_{2}$ uptake enhancement induced by warmer springs. Proceedings of the National Academy of Sciences of the United States of America, 102, 10823-10827.

Arft AM, Walker MD, Gurevitch J et al. (1999) Responses of tundra plants to experimental warming: meta-analysis of the International Tundra Experiment Ecological Monographs, 69, 491-511.
Atkin OK, Edwards EJ, Loveys BR (2000) Response of root respiration to changes in temperature and its relevance to global warming. New Phytologist, 147, 141-154.

Bates JD, Svejcar T, Miller RF, Angell RA (2006) The effects of precipitation timing on sagebrush steppe vegetation. Journal of Arid Environments, 64, 670-697.

Bergner B, Johnstone J, Treseder KK (2004) Experimental warming and burn severity alter soil $\mathrm{CO}_{2}$ flux and soil functional groups in a recently burned boreal forest. Global Change Biology, 10, 1996-2004.

Biasi C, Meyer H, Rusalimova O et al. (2008) Initial effects of experimental warming on carbon exchange rates, plant growth and microbial dynamics of a lichen-rich dwarf shrub tundra in Siberia. Plant and Soil, 307, 191-205.

Breeuwer A, Heijmans, Heijmans MMPD, Robroek BJM, Berendse F (2008) The effect of temperature on growth and competition between Sphagnum species. Oecologia, 156, 155-167.

Breshears DD, Cobb NS, Rich PM et al. (2005) Regional vegetation die-off in response to global-change-type drought. Proceedings of the National Academy of Sciences of the United States of America, 102, 15144-15148.

Bronson DR, Gower ST, Tanner M, Linder S, Van Herk I (2008) Response of soil surface $\mathrm{CO}_{2}$ flux in a boreal forest to ecosystem warming. Global Change Biology, 14, 856-867.

Canadell JG, Le Quéré C, Raupach MR et al. (2007) Contributions to accelerating atmospheric $\mathrm{CO}_{2}$ growth from economic activity, carbon intensity, and efficiency of natural sinks. Proceedings of the National Academy of Sciences, 104, 18866-18870.

Cao M, Woodward FI (1998) Dynamic responses of terrestrial ecosystem carbon cycling to global climate change. Nature, 393, 249-252.

Chen $\mathrm{H}$, Tian $\mathrm{H}$ (2005) Does a general temperature-dependent $Q_{10}$ model of soil respiration exist at biome and global scale? Journal of Integrative Plant Biology, 47, 1288-1302.

Chen S, Lin G, Huang J, He M (2008) Responses of soil respiration to simulated precipitation pulses in semiarid steppe under different grazing regimes. Journal of Plant Ecology, 1, 237-246.

Chen S, Lin G, Huang J, Jenerette GD (2009) Dependence of carbon sequestration on the differential responses of ecosystem photosynthesis and respiration to rain pulses in a semiarid steppe. Global Change Biology, 15, 2450-2461.

Chou WW, Silver WL, Jackson RD, Thompson AW, Allen-Diaz B (2008) The sensitivity of annual grassland carbon cycling to the quantity and timing of rainfall. Global Change Biology, 14, 1382-1394.

Churkina G, Running SW, Schloss ALThe participants of the Potsdam NPP model intercomparison (1999) Comparing global models of terrestrial net primary productivity (NPP): the importance of water availability. Global Change Biology, 5, 46-55.

Ciais P, Reichstein M, Viovy N et al. (2005) Europe-wide reduction in primary productivity caused by the heat and drought in 2003. Nature, 437, 529-533.

Cox PM, Betts RA, Jones CD, Spall SA, Totterdell IJ (2000) Acceleration of global warming due to carbon-cycle feedbacks in a coupled climate model. Nature, $\mathbf{4 0 8}$ 184-187.

Cramer W, Bondeau A, Woodward FI et al. (2001) Global response of terrestrial ecosystem structure and function to $\mathrm{CO}_{2}$ and climate change: results from six dynamic global vegetation models. Global Change Biology, 7, 357-373.

Davidson EA, Belk E, Boone RD (1998) Soil water content and temperature as independent or confounded factors controlling soil respiration in a temperate mixed hardwood forest. Global Change Biology, 4, 217-227.

Davidson EA, Nepstad DC, Ishida FY, Brando PM (2008) Effects of an experimental drought and recovery on soil emissions of carbon dioxide, methane, nitrous oxide, and nitric oxide in a moist tropical forest. Global Change Biology, 14, 2582-2590.

De Dato G, Pellizzaro G, Cesaraccio C et al. (2006) Effects of warmer and drier climate conditions on plant composition and biomass production in a Mediterranean shrubland community. Forest Ecology, 3, 511-526.

De Boeck HJ, Lemmens CMHM et al. (2007) How do climate warming and species richness affect $\mathrm{CO}_{2}$ fluxes in experimental grasslands? New Phytologist, 175, 512 522

De Boeck HJ, Lemmens CMHM, Zavalloni C et al. (2008) Biomass production in experimental grasslands of different species richness during three years of climate warming. Biogeosciences, 5, 585-594.

De Valpine P, Harte J (2001) Plant responses to experimental warming in a Montane Meadow. Ecology, 82, 637-648.

Dormann CF, Woodin SJ (2002) Climate change in the arctic: using plant functional types in a meta-analysis of field experiments. Functional Ecology, 16, 4-17.

Dorrepaal E, Aerts R, Cornelissen JHC, Callaghan TV, Van Logtestijn RSP (2004) Summer warming and increased winter snow cover affect Sphagnum fuscum growth, structure and production in a sub-arctic bog. Global Change Biology, 10, 93-104.

Dukes JS, Chiariello NR, Cleland EE et al. (2005) Responses of grassland production to single and multiple global environmental changes. PLoS Biology, 3, 1829-1837. 
Emmett BA, Beier C, Estiarte M et al. (2004) The response of soil processes to climate change: results from manipulation studies of shrublands across an environmental gradient. Ecosystems, 7, 625-637.

Fay PA, Carlisle JD, Knapp AK, Blair JM, Scott SL (2000) Altering rainfall timing and quantity in a mesic grassland ecosystem: design and performance of rainfall manipulation shelters. Ecosystems, 3, 308-319.

Fay PA, Kaufman DM, Nippert JB, Carlisle JD, Harper CW (2008) Changes in grassland ecosystem function due to extreme rainfall events: implications for responses to climate change. Global Change Biology, 14, 1600-1608.

Friedlingstein P, Cox P, Betts R et al. (2006) Climate-carbon cycle feedback analysis: results from the $C^{4} \mathrm{MIP}$ model intercomparison. Journal of Climate, 19, 3337-3353.

Fung IY, Doney SC, Lindsay K, John J (2005) Evolution of carbon sinks in a changing climate. Proceedings of the National Academy of Sciences of the United States of America, 102, 11201-11206.

Garbulsky MF, Peñuelas J, Papale D et al. (2010) Patterns and controls of the variability of radiation use efficiency and primary productivity across terrestrial ecosystems. Global Ecology Biogeography, 19, 253-267.

Gorissen A, Tietema A, Joosten NN et al. (2004) Climate change affects carbon allocation to the soil in shrublands. Ecosystems, 7, 650-661.

Grime JP, Brown VK, Thompson K et al. (2000) The response of two contrasting limestone grasslands to simulated climate change. Science, 289, 762-765.

Grime JP, Fridley JD, Askew AP, Thompson K, Hodgson JG, Bennett CR (2008) Longterm resistance to simulated climate change in an infertile grassland. Proceedings of the National Academy of Sciences, 105, 10028-10032.

Grogan P, Chapin FS III (2000) Initial effects of experimental warming on above- and belowground components of net ecosystem $\mathrm{CO}_{2}$ exchange in Arctic Tundra. Oecologia, 125, 512-520.

Gunnarsson U, Granberg G, Nilsson M (2004) Growth, production and interspecific competition in Sphagnum: effects of temperature, nitrogen and sulphur treatments on a boreal mire. New Phytologist, 163, 349-359.

Harper CW, Blair JM, Fay PA, Knapp AK, Carlisle JD (2005) Increased rainfall variability and reduced rainfall amount decreases soil $\mathrm{CO}_{2}$ flux in a grassland ecosystem. Global Change Biology, 11, 322-334.

Harpole WS, Potts DL, Suding KN (2007) Ecosystem responses to water and nitrogen amendment in a California grassland. Global Change Biology, 13, 2341-2348.

Harte J, Shaw R (1995) Shifting dominance within a Montane vegetation community: results of a climate-warming experiment. Science, 267, 876-880.

Hartley AE, Neill C, Melillo JM, Crabtree R, Bowles FP (1999) Plant performance and soil nitrogen mineralization in response to simulated climate change in Subarctic Dwarf shrub heath. Oikos, 86, 331-343.

Hartley IP, Heinemeyer A, Evans SP, Ineson P (2007) The effect of soil warming on bulk soil vs. rhizosphere respiration. Global Change Biology, 13, 2654-2667.

Hedges LV, Olkin I (1985) Statistical Methods for Meta-Analysis. Academic Press, London.

Heimann M, Reichstein M (2008) Terrestrial ecosystem carbon dynamics and climate feedbacks. Nature, 451, 289-292.

Heisler-White J, Knapp A, Kelly E (2008) Increasing precipitation event size increases aboveground net primary productivity in a semi-arid grassland. Oecologia, 158, 129-140.

Hobbie SE, Chapin FS III (1998) The response of Tundra plant biomass, aboveground production, nitrogen, and $\mathrm{CO}_{2}$ flux to experimental warming. Ecology, 79, 15261544.

Howard PJA, Howard DM (1979) Respiration of decomposing litter in relation to temperature and moisture: microbial decomposition of tree and shrub leaf litter 2 Oikos, 33, 457-465.

Hungate BA, van Groenigen KJ, Six J et al. (2009) Assessing the effect of elevated $\mathrm{CO}_{2}$ on soil carbon: a comparison of four meta-analyses. Global Change Biology, 15, 20202034.

Huxman TE, Snyder KA, Tissue D et al. (2004) Precipitation pulses and carbon fluxes in semiarid and arid ecosystems. Oecologia, 141, 254-268.

Illeris L, König SM, Grogan P, Jonasson S, Michelsen A, Ro-Poulsen H (2004) Growingseason carbon dioxide flux in a dry subarctic heath: responses to long-term manipulations. Arctic, Antarctic, and Alpine Research, 36, 456-463.

Illeris L, Michelsen A, Jonasson S (2003) Soil plus root respiration and microbial biomass following water, nitrogen, and phosphorus application at a high arctic semi desert. Biogeochemistry, 65, 15-29.

Intergovernmental Panel on Climate Change (IPCC) (2007) Climate change 2007: the physical science basis - summary for policy makers. Contribution of Working Group I to the Fourth Assessment Report of the Intergovernmental Panel on Climate Change. IPCC WGI 4th Assessment Report.
Jonasson S, Michelsen A, Schmidt IK, Nielsen EV (1999) Responses in microbes and plants to changed temperature, nutrient, and light regimes in the arctic. Ecology, 80, 1828-1843.

Jones MH, Fahnestock JT, Walker DA, Walker MD, Welker JM (1998) Carbon dioxide fluxes in moist and dry arctic tundra during the snow-free season: responses to increases in summer temperature and winter snow accumulation. Arctic and Alpine Research, 30, 373-380.

Jónsdóttir IS, Khitun O, Stenström A (2005) Biomass and nutrient responses of a clonal tundra sedge to climate warming. Canadian Journal of Botany, 83, 1608-1621.

Kirschbaum MUF (1995) The temperature dependence of soil organic matter decomposition, and the effect of global warming on soil organic $\mathrm{C}$ storage. Soil Biology and Biochemistry, 27, 753-760.

Knapp AK, Beier C, Briske DD et al. (2008) Consequences of more extreme precipitation regimes for terrestrial ecosystems. Bioscience, 58, 811-821.

Knapp AK, Smith MD (2001) Variation among biomes in temporal dynamics of aboveground primary production. Science, 291, 481-484.

Knorr W, Prentice IC, House JI, Holland EA (2005) Long-term sensitivity of soil carbon turnover to warming. Nature, 433, 298-301.

Kudo G, Suzuki S (2003) Warming effects on growth, production, and vegetation structure of alpine shrubs: a five-year experiment in northern Japan. Oecologia, 135, 280-287.

La Puma IP, Philippi TE, Oberbauer SF (2007) Relating NDVI to ecosystem $\mathrm{CO}_{2}$ exchange patterns in response to season length and soil warming manipulations in arctic Alaska. Remote Sensing of Environment, 109, 225-236.

Lellei-Kovács E, Kovács-Láng E, Kalapos T, Botta-Dukát Z, Barabás S, Beier C (2008) Experimental warming does not enhance soil respiration in a semiarid temperate forest-steppe ecosystem. Community Ecology, 9, 29-37.

Lieth H (1973) Primary production: terrestrial ecosystems. Human Ecology, 1, 303-332.

Lilley J, Bolger T, Gifford R (2001) Productivity of Trifolium subterraneum and Phalaris aquatica under warmer, high $\mathrm{CO}_{2}$ conditions. New Phytologist, 150, 371-383.

Lisa B, Matteo G, Marcello T, Renato G (2007) Responses of subalpine dwarf-shrub heath to irrigation and fertilization. Journal of Vegetation Science, 18, 337-344.

Liu W, Zhang Z, Wan S (2009) Predominant role of water in regulating soil and microbial respiration and their responses to climate change in a semiarid grassland. Global Change Biology, 15, 184-195.

Loik ME, Breshears DD, Lauenroth WK, Belnap J (2004) A multi-scale perspective of water pulses in dryland ecosystems: climatology and ecohydrology of the Western USA. Oecologia, 141, 269-281.

Lucht W, Prentice IC, Myneni RB et al. (2002) Climatic control of the high-latitude vegetation greening Trend and Pinatubo effect. Science, 296, 1687-1689.

Luo Y, Gerten D, Le Maire G et al. (2008) Modeled interactive effects of precipitation, temperature, and $\left[\mathrm{CO}_{2}\right]$ on ecosystem carbon and water dynamics in different climatic zones. Global Change Biology, 14, 1986-1999.

Luo Y, Sherry R, Zhou X, Wan S (2009) Terrestrial carbon-cycle feedback to climate warming: experimental evidence on plant regulation and impacts of biofuel feedstock harvest. Global Change Biology Bioenergy, 1, 62-74.

Luo Y, Wan S, Hui D, Wallace LL (2001) Acclimatization of soil respiration to warming in a tall grass prairie. Nature, 413, 622-625.

Marchand FL, Nijs I, Boeck HJd, Kockelbergh F, Mertens S, Beyens L (2004) Increased turnover but little change in the carbon balance of high-arctic tundra exposed to whole growing season warming. Arctic, Antarctic, and Alpine Research, 36, 298-307.

McCulley RL, Boutton TW, Archer SR (2007) Soil respiration in a subtropical Savanna Parkland: response to water additions. Soil Science Society of America Journal, 71, 820-828.

McHale PJ, Mitchell MJ, Bowles FP (1998) Soil warming in a northern hardwood forest: trace gas fluxes and leaf litter decomposition. Canadian Journal of Forest Research, 28, 1365-1372.

Melillo JM, Steudler PA, Aber JD et al. (2002) Soil warming and carbon-cycle feedbacks to the climate system. Science, 298, 2173-2176.

Mertens S, Nijs I, Heuer M, Kockelbergh F, Beyens L, Kerckvoorde Av, Impens I (2001) Influence of high temperature on end-of-season tundra $\mathrm{CO}_{2}$ exchange. Ecosystems, 4, 226-236.

Miranda JdD, Padilla FM, Lázaro R, Pugnaire FI (2009) Do changes in rainfall patterns affect semiarid annual plant communities? Journal of Vegetation Science, 20, 269-276.

Mirzaei H, Kreyling J, Hussain MZ, Li Y, Tenhunen J, Beierkuhnlein C, Jentsch A (2008) A single drought event of 100-year recurrence enhances subsequent carbon uptake and changes carbon allocation in experimental grassland communities. Journal of Plant Nutrition and Soil Science, 171, 681-689. 
Nemani RR, Keeling CD, Hashimoto H et al. (2003) Climate-driven increases in global terrestrial net primary production from 1982 to 1999. Science, 300, 1560-1563.

Niinistö SM, Silvola J, Kellomäki S (2004) Soil $\mathrm{CO}_{2}$ efflux in a boreal pine forest under atmospheric $\mathrm{CO}_{2}$ enrichment and air warming. Global Change Biology, 10, 13631376.

Niu S, Wu M, Han Y, Xia J, Li L, Wan S (2008) Water-mediated responses of ecosystem carbon fluxes to climatic change in a temperate steppe. New Phytologist, 177, 209219.

Oberbauer SF, Tweedie CE, Welker JM et al. (2007) Tundra $\mathrm{CO}_{2}$ fluxes in response to experimental warming across latitudinal and moisture gradients. Ecological Monographs, 77, 221-238.

Ogaya R, Peñuelas J (2007) Tree growth, mortality, and above-ground biomass accumulation in a holm oak forest under a five-year experimental field drought. Plant Ecology, 189, 291-299.

Olszyk DM, Johnson MG, Tingey DT et al. (2003) Whole-seedling biomass allocation, leaf area, and tissue chemistry for Douglas-fir exposed to elevated $\mathrm{CO}_{2}$ and temperature for 4 years. Canadian Journal of Forest Research, 33, 269-278.

Patrick L, Cable J, Potts D et al. (2007) Effects of an increase in summer precipitation on leaf, soil and ecosystem fluxes of $\mathrm{CO}_{2}$ and $\mathrm{H}_{2} \mathrm{O}$ in a sotol grassland in Big Bend National Park, Texas. Oecologia, 151, 704-718.

Peñuelas J, Filella I (2001) Phenology: responses to a warming world. Science, 294, 793 795.

Peñuelas J, Prieto P, Beier C et al. (2007) Response of plant species richness and primary productivity in shrublands along a north-south gradient in Europe to seven years of experimental warming and drought: reductions in primary productivity in the heat and drought year of 2003. Global Change Biology, 13, 2563-2581.

Press MC, Potter JA, Burke MJW, Callaghan TV, Lee JA (1998) Responses of a Subarctic Dwarf shrub heath community to simulated environmental change. The Journal of Ecology, 86, 315-327.

Risch AC, Frank DA (2007) Effects of increased soil water availability on grassland ecosystem carbon dioxide fluxes. Biogeochemistry, 86, 91-103.

Robroek BJM, Schouten MGC, Limpens J, Berendse F, Poorter H (2009) Interactive effects of water table and precipitation on net $\mathrm{CO}_{2}$ assimilation of three cooccurring Sphagnum mosses differing in distribution above the water table. Global Change Biology, 15, 680-691.

Rosenberg MS, Adams DC, Gurevitch J (2000) MetaWin: Statistical Software for MetaAnalysis. Version 2. Sinauer Associates, Sunderland, MA.

Rustad LE, Campbell JL, Marion GM et al. (2001) A meta-analysis of the response of soil respiration, net nitrogen mineralization, and aboveground plant growth to experimental ecosystem warming. Oecologia, 126, 543-562.

Rustad LE, Fernandez IJ (1998) Experimental soil warming effects on CO[sub 2] and $\mathrm{CH}$ [sub 4] flux from a low elevation spruce-fir forest soil in Maine, USA. Global Change Biology, 4, 597-605.

Saleska SR, Miller SD, Matross DM et al. (2003) Carbon in Amazon forests: unexpected seasonal fluxes and disturbance-induced losses. Science, 302, 1554-1557.

Saleska SR, Shaw MR, Fischer ML, Dunne JA, Still CJ, Holman ML, Harte J (2002) Plant community composition mediates both large transient decline and predicted longterm recovery of soil carbon under climate warming. Global Biogeochemical Cycles, 16, 1055, doi: 10.1029/2001GB001573.

Sardans J, Penuuelas J, Estiarte M, Prieto P (2008) Warming and drought alter C and N concentration, allocation and accumulation in a Mediterranean shrubland. Global Change Biology, 14, 2304-2316.

Schindlbacher A, Zechmeister-Boltenstern S, Jandl R (2009) Carbon losses due to soil warming: do autotrophic and heterotrophic soil respiration respond equally? Global Change Biology, 15, 901-913.

Schleser GH (1982) The response of $\mathrm{CO}_{2}$ evolution from soils to global temperature changes. Zeitschrift Naturforschung, 37, 287-291.

Scurlock JMO, Johnson K, Olson RJ (2002) Estimating net primary productivity from grassland biomass dynamics measurements. Global Change Biology, 8, 736-753.

Shaver GR, Canadell J, Chapin FS III et al. (2000) Global warming and terrestrial ecosystems: a conceptual framework for analysis. Bioscience, 50, 871-882.

Sherry RA, Weng E, Arnone JA III et al. (2008) Lagged effects of experimental warming and doubled precipitation on annual and seasonal aboveground biomass production in a tallgrass prairie. Global Change Biology, 14, 2923-2936.

Siemann E, Rogers WE, Grace JB (2007) Effects of nutrient loading and extreme rainfall events on coastal tallgrass prairies: invasion intensity, vegetation responses, and carbon and nitrogen distribution. Global Change Biology, 13, 2184-2192.
Silver WL, Jackson RD, Allen-Diaz B (2005) Soil carbon dynamics of California grasslands under altered soil moisture regimes. Kearney Foundation of Soil Science Final Report, pp. 1-14.

Solomon S, Qin D, Manning M et al (eds) (2007) IPCC, 2007: Climate Change 2007: The Physical Science Basis. Contribution of Working Group I to the Fourth Assessment Report of the Intergovernmental Panel on Climate Change. Cambridge University Press, Cambridge, UK.

Sotta ED, Veldkamp E, Schwendenmann L et al. (2007) Effects of an induced drought on soil carbon dioxide $\left(\mathrm{CO}_{2}\right)$ efflux and soil $\mathrm{CO}_{2}$ production in an Eastern Amazonian rainforest, Brazil. Global Change Biology, 13, 2218-2229.

Sitch S, Huntingford C, Gedney N et al. (2008) Evaluation of the terrestrial carbon cycle, future plant geography and climate-carbon cycle feedbacks using five Dynamic Global Vegetation Models (DGVMs). Global Change Biology, 14, 2015-2039.

Sullivan PF, Arens SJT, Chimner RA, Welker JM (2008) Temperature and microtopography interact to control carbon cycling in a high arctic fen. Ecosystems, 11, 61-76.

Thompson K, Grime JP, Hillier SH, Askew AP, Corker D, Kielty JP (2000) Predicting the response of limestone grassland to climate change. Aspects of Applied Biology, 58, 329-336.

Tingey DT, Lee EH, Phillips DL, Rygiewicz PT, Waschmann RS, Johnson MG, Olszyk $\mathrm{DM}$ (2007) Elevated $\mathrm{CO}_{2}$ and temperature alter net ecosystem $\mathrm{C}$ exchange in a young Douglas fir mesocosm experiment. Plant, Cell and Environment, 30, 1400-1410.

Updegraff K, Bridgham SD, Pastor J, Weishampel P, Harth C (2001) Response of $\mathrm{CO}_{2}$ and $\mathrm{CH}_{4}$ emissions from peatlands to warming and water table manipulation. Ecological Applications, 11, 311-326.

Wan S, Hui D, Wallace L, Luo Y (2005) Direct and indirect effects of experimental warming on ecosystem carbon processes in a tallgrass prairie. Global Biogeochemical Cycles, 19, GB2014, doi:10.1029/2004GB002315.

Wan S, Norby RJ, Pregitzer KS, Ledford J, O'Neill EG (2004) $\mathrm{CO}_{2}$ enrichment and warming of the atmosphere enhance both productivity and mortality of maple tree fine roots. New Phytologist, 162, 437-446.

Wan SQ, Norby RJ, Ledford J, Weltzin JF (2007) Responses of soil respiration to elevated $\mathrm{CO}_{2}$, air warming, and changing soil water availability in a model oldfield grassland. Global Change Biology, 13, 2411-2424.

Welker JM, Brown KB, Fahnestock JT (1999) $\mathrm{CO}_{2}$ flux in arctic and alpine dry tundra: comparative field responses under ambient and experimentally warmed conditions. Arctic, Antarctic, and Alpine Research, 31, 272-277.

Welker JM, Fahnestock JT, Henry GHR, O'Dea KW, Chimner RA (2004) $\mathrm{CO}_{2}$ exchange in three Canadian high arctic ecosystems: response to long-term experimental warming. Global Change Biology, 10, 1981-1995.

Weltzin JF, Pastor J, Harth C, Bridgham SD, Updegraff K, Chapin CT (2000) Response of bog and fen plant communities to warming and water-table manipulations. Ecology, 81, 3464-3478.

Woodwell GM, Mackenzie FT, Houghton RA, Apps M, Gorham E, Davidson E (1998) Biotic feedbacks in the warming of the earth. Climatic Change, 40, 495-518.

Wookey PA, Robinson CH, Parsons AN, Welker JM, Press MC, Callaghan TV, Lee JA (1995) Environmental constraints on the growth, photosynthesis and reproductive development of Dryas octopetala at a high arctic polar semi-desert, Svalbard. Oecologia, 102, 478-489.

Xia J, Niiu S, Wan S (2009) Response of ecosystem carbon exchange to warming and nitrogen addition during two hydrologically contrasting growing seasons in a temperate steppe. Global Change Biology, 15, 1544-1556.

Xiao C, Janssens IA, Liu P, Zhou Z, Sun OJ (2007) Irrigation and enhanced soil carbon input effects on below-ground carbon cycling in semiarid temperate grasslands. New Phytologist, 174, 835-846.

Zavaleta ES, Shaw MR, Chiariello NR, Thomas BD, Cleland EE, Field CB, Mooney HA (2003) Grassland responses to three years of elevated temperature, $\mathrm{CO}_{2}$, precipitation, and $\mathrm{N}$ deposition. Ecological Monographs, 73, 585-604.

Zhou X, Sherry RA, An Y, Wallace LL, Luo Y (2006) Main and interactive effects of warming, clipping, and doubled precipitation on soil $\mathrm{CO}_{2}$ efflux in a grassland ecosystem. Global Biogeochemical Cycles, 20, GB1003, doi: 10.1029/ 2005GB002526.

Zhou X, Wan S, Luo Y (2007) Source components and interannual variability of soil $\mathrm{CO}_{2}$ efflux under experimental warming and clipping in a grassland ecosystem. Global Change Biology, 13, 761-775.

Zogg GP, Zak DR, Ringelberg DB, MacDonald NW, Pregitzer KS, White DC (1997) Compositional and functional shifts in microbial communities due to soil warming. Soil Science Society of America Journal, 61, 475-481. 